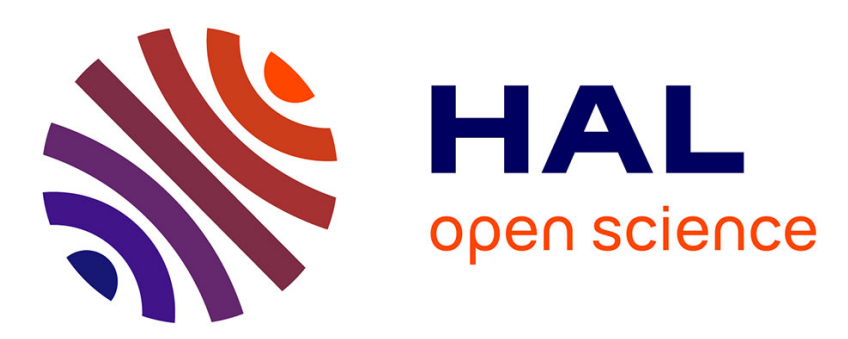

\title{
Quasi-Gaussian probability density function of sea wave slopes from near nadir $\mathrm{Ku}$-band radar observations Ping Chen, Gang Zheng, Danièle Hauser, Fei Xu
}

\section{To cite this version:}

Ping Chen, Gang Zheng, Danièle Hauser, Fei Xu. Quasi-Gaussian probability density function of sea wave slopes from near nadir Ku-band radar observations. Remote Sensing of Environment, 2018, 217, pp.86 - 100. 10.1016/j.rse.2018.07.027 . insu-01856646

\section{HAL Id: insu-01856646 https://hal-insu.archives-ouvertes.fr/insu-01856646}

Submitted on 17 Nov 2019

HAL is a multi-disciplinary open access archive for the deposit and dissemination of scientific research documents, whether they are published or not. The documents may come from teaching and research institutions in France or abroad, or from public or private research centers.
L'archive ouverte pluridisciplinaire HAL, est destinée au dépôt et à la diffusion de documents scientifiques de niveau recherche, publiés ou non, émanant des établissements d'enseignement et de recherche français ou étrangers, des laboratoires publics ou privés. 


\title{
Quasi-Gaussian Probability Density Function of sea wave slopes from Near Nadir Ku-Band Radar Observations
}

\author{
Ping Chen ${ }^{1.4}$,Gang Zheng ${ }^{2}$,Danièle Hauser ${ }^{3}$, Fie Xu $u^{1.4}$ * \\ ${ }^{1}$ School of Electronic Information and Communications, Huazhong University of Science \& Technology, \\ Wuhan 430074, Hubei, China \\ ${ }^{2}$ Second Institute of Oceanography, State Oceanic Administration, Hangzhou \\ ${ }^{3}$ Universitè de Versailles Saint-Quentin-en-Yvelines, Universite Paris 06, CNRS, LATMOS-IPSL, \\ Guyancourt, France \\ ${ }^{4}$ Science and Technology on Multi-spectral Information Processing Laboratory
}

\begin{abstract}
Although in most circumstances, sea wave slope probability density function (PDF) is expressed as Gaussian distribution, there is evidence that it follows quasi-Gaussian distribution, which can be represented by Gram-Charlier series to fourth order. All the statistical parameters of slope PDF have previously been derived by using optical methods in specular conditions, and values and relationships with surface parameters have been presented in the literature. However they may not be relevant at microwave wavelengths due to diffraction effects. Up to now, sea surface slope PDF consistent with ocean microwave remote sensing is not known yet. So it is important to establish the parameter models of quasi-Gaussian slope PDF compatible with radar application. In this paper, based on the backscattering coefficients from the Ku-band space-borne radar Precipitation Radar (PR) data, all the parameters of the quasi-Gaussian slope PDF are inverted using a so-called "GO4" (Boisot et al. (2015)) model with a two-dimensional (2-D) non-linear least square fit on the backscattering coefficients. We also establish the empirical formulae relating the statistical parameters of the quasi-Gaussian sea slope PDF with wind speed, which may be used for ocean Ku-band radar application.

The proposed empirical formulae are compared to the Cox and Munk (1954)-CM slope parameter model: the results confirm that the slope variance in upwind and crosswind
\end{abstract}

\footnotetext{
${ }^{*}$ Correpondence author: Fei Xu, Email: xufei_hust@163.com
} 
directions as well as the skewness coefficients exhibit intermediate values between the CM slope parameters of clean surface and slick surface cases. The coefficients of peakedness are just in the range of the CM slope peakedness parameter values.

The impacts of wave conditions (swell or wind sea) on slope PDF parameters are also studied. The results show that in most wind speed conditions, the presence of swell increases the skewness coefficients, while it decreases the peakedness coefficients.

Keywords: slope probability density function of sea surface, Ku-band radar, near-nadir radar cross-sections, approximate scattering model

\section{Introduction}

Ocean surface waves are a topic of active research within physical oceanography, due to their role in the coupled ocean/atmosphere system and to their impact on various society sectors (national defense, navigation, shipbuilding and offshore industry...). The distribution of wave slopes is an important statistical tool in describing ocean surface waves, because it is related to a number of physical processes which occur at or near the air-sea interface, such as the dynamics including wave breaking and the nonlinear energy transfer between wavenumbers, which is a strong function of both the energy-containing and high frequency waves (see e.g. Longuet-Higgins (1978); Om (1985); Resio and Perrie (1991)). The distribution of wave slopes is an important quantity in the processes of wave generation, wave growth or dissipation as well as air/sea interactions.

Due to the random nature of sea wave, the slope probability density function (PDF) is usually used to represent the wave slopes. The scattering of acoustic and electromagnetic waves in the optical or microwave domains is closely related to the wave slope PDF.

In the optical domain, the sea surface scattering can be considered as specular, which leads to a linear relationship with the slope PDF based on geometrical optics (GO) analytical approximations. There are historical and more recent results presented in the literature Jackson et al. (1992); Cox and Munk $(1954,1956)$ which are based on this property to estimate the surface slope PDF at optical wavelengths. Based on the analysis of sun glitter on 
the sea surface, Cox and Munk (1954) used the geometrical optics approximation combined with the assumption that sea surface slope PDF is quasi-Gaussian and can be expressed by a Gram-Charlier expansion up to the fourth order, where the slope variances, skewness coefficients and peakedness coefficients are included. They established empirical formulae relating the seven parameters of the slope PDF approximated by the Gram-Charlier expansion, to wind speed. Brèon and Henriot (2006) used visible light reflection data provided by POLDER multiple angular radiometer carried on the ADEOS-1 satellite and wind data from the NSCAT wind scatterometer to invert the same parameters under various wind speed conditions and to revisit the empirical formulae proposed by Cox and Munk. In these studies, it was assumed that the slope PDF is only related to wind speed.

However, these results obtained from optical measurements cannot be transposed directly in the application of ocean microwave remote sensing because of the diffraction effects at wavelengths longer than optical ones. So it is important to establish the parameter models of quasi-Gaussian slope PDF for radar application. This is the aim of our work.

In the microwave domain, at low incidence (i.e., near-nadir incidence) the sea surface scattering can be considered as quasi-specular, the geometrical optics (GO) approximation still holds if one considers the diffraction-modified Fresnel reflectivity (Tsang and Kong (2001)) and the slope PDF of surfaces waves only for waves longer than the diffraction limit (Jackson et al. (1992); Barrick (1968)). This motivated the introduction and use of the notion of radar-filtered slope statistics by several authors Jackson et al. (1992); Tsang and Kong (2001); Barrick (1968); Hauser et al. (2008); Boisot et al. (2015); Freilich and Vanhoff (2003); Chu et al. (2012a). The following paragraphs give the review of these studies. In Jackson et al. (1992), Freilich and Vanhoff (2003) such filtered sea slope PDF is assumed Gaussian and filtered slope variances is studied, while in Hauser et al. (2008), Chu et al. (2012a) such sea slope PDF is assumed quasi-Gaussian, however, due to the limits of both the scattering model and the inversion method, not all parameters in the slope PDF can be obtained. This furthermore motivates us to study the approximation scattering model at low incidence with high accuracy, as well as the inversion method, then to find all the seven parameters in a 
quasi-Gaussian filtered" slope PDF, and establish the parameter models of quasi-Gaussian slope PDF for radar application.

Results on the slope PDF estimated from microwave observations have also been presented in the literature Boisot et al. (2015); Freilich and Vanhoff (2003); Tsang and Kong (2001); Hauser et al. (2008). With the assumption of a Gaussian slope PDF and observations very close to nadir it is admitted that the filtering occurs below three to five times the radar wavelength. However, this may vary with incidence range and with roughness conditions.

With the assumption of isotropy (the slope variance in upwind equals that in crosswind) and a Gaussian slope PDF, Jackson et al. (1992) averaged the backscatter coefficients at different azimuth from the Ku-band airborne-spectrometer ROWS (incidences of 0-20 ${ }^{\circ}$ ), and derived the slope variances from a one-dimensional (1-D) inversion method. He established empirical formulae for the variation of slope variances with wind speed applicable for $\mathrm{Ku}-$ band observations. The same method was used later (Freilich and Vanhoff (2003)) on a larger data set by using Tropical Rainfall Mapping Mission (TRMM) data of Precipitation radar $(\mathrm{PR})$ in the $0-18^{\circ}$ incidence range co-located with wind estimates from the TRMM microwave Imager.

Hauser et al. (2008) analyzed 2-D backscattering coefficients (as a function of incidence and azimuth) at C-band from the airborne-spectrometer STORM to derive the slope variances in upwind and crosswind directions as well as a peakedness parameter based on the compound model of slope PDF by Chu et al. (2012a). However, because the inversion was applied independently for each azimuth observation, the skewness coefficients, which are related to the anisotropic properties of slope PDF, were not studied.

Chu et al. (2012a) used the backscattering coefficients from the Precipitation Radar (PR) of Tropical Rainfall Mapping Mission (TRMM) co-located with wind information from buoys to invert the slope variance in upwind and crosswind directions and two skewness coefficients under various wind speeds. They used the heuristic inversion method also used by Cox and Munk. Their results show that the asymmetry of backscattering between downwind and 
upwind at low incidence is caused by the skewness of wave slope PDF. However, three coefficients of peakedness have not been estimated in their study. Therefore, the complete relationships between the seven parameters of the quasi-Gaussian sea wave slope PDF and wind speed have not been established for microwave band until now.

Besides optical and microwave methods, Vandemark et al. (2004) estimated slope PDF by using direct range measurements with an airborne laser, but the approach provides information only in a non-directional sense, and for waves longer than about $2 \mathrm{~m}$ in wavelength. Shaw and Churnside (1997); Hwang and Wang (2004) and Hwang (2005) made in situ spectral measurements of ocean waves from a free-drifting buoy and estimated the variance of the slope PDF of ocean waves whose wavelength are in the range of about 0.02-6 m.

In the references mentioned here-above the inversion of the slope PDF from data set in the microwave band is based on the Quasi-Specular (QS) model, i.g., the GO scattering model with filtered slope statistic parameters and the diffraction-modified Fresnel reflectivity. However, QS model accuracy is only of the order of several percent in Hauser et al. (2008) at low incidence angles, if compared with the Physical Optics model (PO), which is considered as the reference model at near-nadir incidences. For the case of Gaussian slope PDF, the QS model accuracy does not affect significantly the inversion results on slope variances because the inversion of Gaussian slope PDF is a kind of linear inversion. In contrast, for the case of quasi-Gaussian slope PDF where the aim is to invert higher order parameters of the slope statistics, such as peakedness and skewness coefficients, the effect of curvature must be taken into account by Bringer et al. (2012); Boisot et al. (2015). So QS model accuracy is not enough for this case since the curvature effect is ignored in QS model.

Bringer et al. (2012) developed a GO4 model by using the 4th order expansion (instead of $2^{\text {nd }}$ order expansion in GO) of the structure function which appears in the Kirchhoff integral of the PO model to take the effect of curvature into account. In their model, both slope and curvature parameters are considered as total and the model agrees well in the first few degrees of incidence with PO. However, ignoring the filtering effect on slope and curvature variances for microwave band results in a decrease of model accuracy as the incidence angle 
increases. Boisot et al. (2015) improved the interpretations for the parameters of the GO4, i.g., only slope parameters are considered total while the curvature parameters is regarded as filtered. With the improvement, the accuracy of GO4 in Boisot et al. (2015) is increased relative to that of the former version of GO4 presented in Bringer et al. (2012).

In this paper, we use the same model GO4 and we will show in a first part (Section 2) that in opposite to the results of Boisot et al. (2015) and Bringer et al. (2012), we must invoke parameters of the surface slope PDF filtered at a certain scale to reproduce with a high accuracy the PO model. In opposite to Boisot et al. (2015) and Freilich and Vanhoff (2003) our approach takes into account the anisotropic nature of the surface (variations with azimuth angle). Then, using the TRMM/Precipitation Radar (PR) data set co-located with buoy measurements, the dependence of the backscattering coefficients with both incidence and azimuth angles are analyzed. By applying a non-linear fit of the GO4 model to the observations, all the seven coefficients of the Gram-Charlier expansion of a quasi-Gaussian slope PDF are inverted under different wind speeds; furthermore, empirical formulae relating each of the seven parameters with wind speed are proposed for the first time for Ku-band.

The paper is organized as follows. In section 2.1, we introduce the scattering model (GO4) used for estimating the parameters of the quasi-Gaussian slope PDF from the normalized radar cross-sections. In Section 2.2, we analyze the results of GO4 inversion applied on backscatter simulations. The reference of the simulation are normalized radar crosssections calculated from the PO model and a standard surface description (wave spectrum from Elfouhaily et al. (1997) in a wind sea case, and mixed sea case with wind sea and swell). This part allows us to assess the range of incidence and wind conditions in which the differences between GO4 and PO are minimum. Simulations are also used to estimate the cut-off wavelength of the inverted parameters. Section 3 briefly describes the data set used in the present analysis (PR observations from the TRMM satellite). Section 4 presents the results obtained from the inversion of the PR data set, and provides comparison with the Cox and Munk (1954)-CM- model. Then, empirical formulas for the seven effective parameters of slope PDF with wind speed are summarized. The main results are summarized in 
the conclusion.

\section{Slope PDF Inversion Model and Method}

\subsection{Scattering Model}

At near-nadir incidence angles, the PO scalar approximation is considered accurate enough as long as polarization effects remain negligible, that is in the first $20-25^{\circ}$ incidence away from nadir by Thompson et al. (2005). In the following, PO is referred to as the reference model, and other models mentioned are all approxiamation models. Indeed, to overcome the limitations of the classical GO model, as well as QS model, Boisot et al. (2015) and Bringer et al. (2012) proposed an alternative approximation called GO4, to take into account possible deviation of the surface from the approximate tangent plane. The main idea proposed in Boisot et al. (2015) and Bringer et al. (2012) was to make use of the 4th order expansion (instead of $2^{\text {nd }}$ order expansion in GO) of the structure function which appears in the Kirchhoff integral of the PO model (Boisot et al. (2015)). Both the slope and curvature variances in GO4 in Bringer et al. (2012) are total. Compared with GO4 in Bringer et al. (2012), the improvement of GO4 in Boisot et al. (2015) is that the curvature variances are considered as filtered. GO4 in Boisot et al. (2015) express the normalized radar cross-section (NRCS) in the isotropic case as Boisot et al. (2015):

$$
\begin{aligned}
\sigma_{G O 4}^{0}(\theta, \varphi)= & \frac{|R|^{2}}{m s s} \sec ^{4}(\theta) \exp \left(-\frac{\tan ^{2}(\theta)}{m s s}\right) \times \\
& {\left[1+\frac{m s c_{e}}{16 K^{2} m s s^{2} \cos ^{2} \theta}\left(\frac{\tan ^{4}(\theta)}{m s s^{2}}-4 \frac{\tan ^{2}(\theta)}{m s s}+2\right)\right] }
\end{aligned}
$$

where $R$ is the Fresnel reflectivity, $\theta$ the incidence angle, mss the total mean square slope and $m s c_{e}$ the filtered mean square curvature of the sea surface. This equation was derived in Boisot et al. (2015) by considering that the msc value is relative to a filtered surface and $m s c_{e}$ can be determined with the use of additional PO NRCS at incidence $0^{\circ}$ (and only at this incidence). 
In the anisotropic case, Eq.(1) becomes:

$$
\begin{gathered}
\sigma_{G O 4}^{0}(\theta, \varphi)=\frac{|R|^{2}}{2 \sqrt{m s s x} \sqrt{m s s y}} \sec ^{4}(\theta) \exp \left(-\frac{1}{2}\left(X^{2}+Y^{2}\right)\right) \times \\
\left\{1+\frac{1}{96 K^{2} \cos ^{2} \theta}\left[\begin{array}{l}
\frac{6 m s c x y_{e}}{m s s x \cdot m s s y} H_{2}(X) H_{2}(Y) \\
+\frac{m s c x_{e}}{m s s x^{2}} H_{4}(X)+\frac{m s c y_{e}}{m s s y^{2}} H_{4}(Y)
\end{array}\right]\right\}
\end{gathered}
$$

where $m s s x$ and mssy are total mean square slopes in two orthogonal directions, $m s c x$, $m s c y$, and $m s c x y$ are directional curvatures, $H_{n}$ is the Hermitte polynomials of order $n$ and $X$ and $Y$ defined as:

$$
H_{n}(u)=(-1)^{n} e^{\frac{u^{2}}{2}} \frac{d^{n}}{d u^{n}} e^{-\frac{u^{2}}{2}}, X=\frac{\tan \theta \cos \varphi}{\sqrt{m s s x_{e}}}, Y=\frac{\tan \theta \sin \varphi}{\sqrt{m s s y_{e}}}
$$

Here we use the same models, but instead of imposing this constraint, $m s s, m s c$ and $R$ in Eq.(1) and mssx, mssy, mscx, mscy, mscxy, and $R$ in Eq.(2) are obtained by directly fitting Eq.(1) or Eq.(2) to PO $\sigma^{0}$ over a chosen incidence range. We will show below (see section 2.2 ), that in fact the variable mss, msc, mssx, mssy, mscx, mscy and mscxy obtained by such fitting are relative to the filtered surface, and $R$ is also a diffraction-modified coefficient.

We recall by comparison, that the QS model under the assumption of Gaussian statistics of the surface writes:

$$
\sigma_{Q S}^{0}(\theta, \varphi)=\frac{\left|R_{e}\right|^{2}}{2 \sqrt{m s s x_{e}} \sqrt{m s s y_{e}}} \sec ^{4}(\theta) \exp \left(-\frac{1}{2}\left(X^{2}+Y^{2}\right)\right)
$$

Where both $m s s x_{e}$ and $m s s y_{e}$ are filtered mean square slopes, and $R_{e}$ is also a diffractionmodified coefficient.

In Eq.(1-3), the surface was considered as Gaussian. In reality, as shown in Cox and Munk $(1956,1954)$, the ocean surface is a weakly non-Gaussian surfac, and the Gram-Charlier series developed to the fourth order can be used to express such a quasi-Gaussian sea slope 
PDF:

$$
\begin{aligned}
p(X, Y)= & \frac{1}{2 \pi \sqrt{m s s x} \sqrt{m s s y}} \exp \left(-\frac{1}{2}\left(X^{2}+Y^{2}\right)\right) \times\left[1+\frac{\lambda_{12}}{2} H_{2}(Y) H_{1}(X)+\right. \\
& \left.\frac{\lambda_{30}}{6} H_{3}(X)+\frac{\lambda_{22}}{4} H_{2}(Y) H_{2}(X)+\frac{\lambda_{40}}{24} H_{4}(X)+\frac{\lambda_{04}}{24} H_{4}(Y)\right]
\end{aligned}
$$

Where $\lambda_{12}, \lambda_{30}$ are skewness coefficients for sea surface slope, $\lambda_{22}, \lambda_{40}$ and $\lambda_{04}$ are peakedness coefficients for sea surface slope.

The skewness and kurtosis parameters are related to the structure function of the 3rd and 4th order in PO by Thompson et al. (2005). Using the definition of the structure functions and expanding them to the third order and the fourth order, they can be approximated for small arguments by:

$$
S_{3}(x, y) \approx \lambda_{30} m s s x^{3 / 2} x^{3}+3 \lambda_{12} m s s x \sqrt{m s s y} x y^{2}
$$

$$
S_{4}(x, y) \approx \lambda_{40} m s s x^{2} x^{4}+\lambda_{04} m s s y^{2} y^{4}+6 \lambda_{22} m s s x \cdot m s s y \cdot x^{2} y^{2}
$$

where the dimensionless coefficients $\lambda_{m n}$ are defined by

$$
\lambda_{m n}=\frac{\left\langle\left(\partial_{x} \eta\right)^{m}\left(\partial_{y} \eta\right)^{n}\right\rangle}{\left\langle\left(\partial_{x} \eta\right)^{2}\right\rangle^{m / 2}\left\langle\left(\partial_{y} \eta\right)^{2}\right\rangle^{n / 2}}
$$

With these assumptions, Boisot et al. (2015) expressed the NCRS of the GO4 model for quasi-Gaussian sea surface. They obtained the following equation:

$$
\left.\begin{array}{c}
\sigma_{G O 4}^{0}(\theta, \varphi)=\frac{|R|^{2}}{2 \sqrt{m s s x} \sqrt{m s s y}} \sec ^{4}(\theta) \exp \left(-\frac{1}{2}\left(X^{2}+Y^{2}\right)\right) \times \\
\left.1+\frac{1}{24 Q_{z}^{2}}\left[\begin{array}{l}
6\left(\frac{m s c x y_{e}}{m s s x \cdot m s s y}+\lambda_{22} Q_{z}^{2}\right) H_{2}(X) H_{2}(Y) \\
+\left(\frac{m s c x_{e}}{m s s x^{2}}+\lambda_{40} Q_{z}^{2}\right) H_{4}(X) \\
+\left(\frac{m s c y_{e}}{m s s y^{2}}+\lambda_{04} Q_{z}^{2}\right) H_{4}(Y)
\end{array}\right]\right\} \\
+\frac{1}{6}\left[3 \lambda_{12} H_{1}(X) H_{2}(Y)+\lambda_{30} H_{3}(X)\right]
\end{array}\right\}
$$

where $Q_{z}$ is twice the radar wavenumber projected in the vertical direction.

In the development of Boisot et al. (2015) the mean square slope parameters are supposed to be non-filtered parameters whereas the curvature parameters are filtered parameters. We 
will see in section below that in fact, all parameters in Eq.(8) related to slope and curvature are filtered parameters, and that $R$ is a diffraction-modified reflection coefficient.

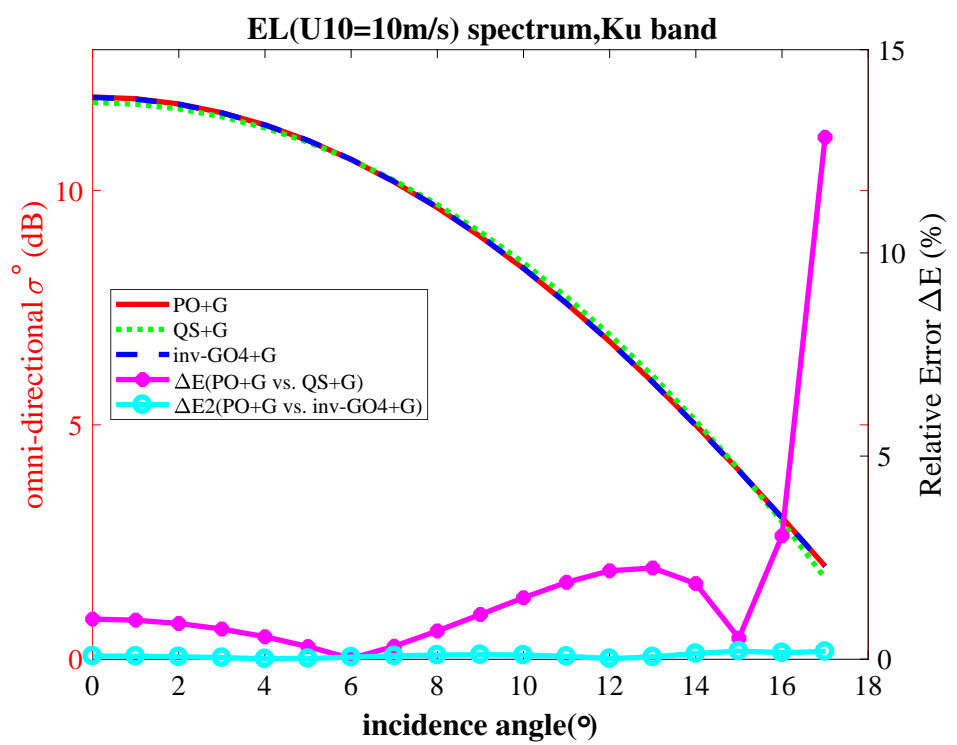

Fig. 1. $\sigma^{\circ}(\theta)$ (in $\mathrm{dB}$ ) as a function of $\theta$ with the $\mathrm{PO}$ model (red line) for a $10 \mathrm{~m} / \mathrm{s}$ wind speed, using EL spectrum, for Ku-band, and after averaging $\sigma^{\circ}(\theta, \varphi)$ in all azimuths. The results from the fit of the GO4 and QS model are shown with the dotted blue and green lines, denoted by inv-GO4 and QS, respectively. Curve with dots represent relative errors between PO and inv-GO4 (cyan), between PO and QS (magenta), respectively.

We recall here for comparison with Eq.(8), that the QS model in the case of a quasiGaussian surface writes:

$$
\begin{aligned}
\sigma_{Q S}^{0}(\theta, \varphi) & =\frac{\left|R_{e}\right|^{2}}{2 \sqrt{m s s x_{e}} \sqrt{m s s y_{e}}} \sec ^{4}(\theta) \exp \left(-\frac{1}{2}\left(X^{2}+Y^{2}\right)\right) \times \\
& {\left[1+\frac{\lambda_{12}}{2} H_{1}(X) H_{2}(Y)+\frac{\lambda_{30}}{6} H_{3}(X)+\frac{\lambda_{22}}{4} H_{2}(Y) H_{2}(X)+\frac{\lambda_{40}}{24} H_{4}(X)+\frac{\lambda_{04}}{24} H_{4}(Y)\right] }
\end{aligned}
$$

\subsection{Conditions for GO4 inversion determined by simulations}

Before using GO4 to invert real data, we tested the ability of the model to reproduce, in the Gaussian case, the PO physical model results and we compared the results with $\sigma^{\circ}$ of QS model in Jackson et al. (1992) (as well as Freilich and Vanhoff (2003), Chu et al. (2012a), Hauser et al. (1992), Jackson et al. (1985), Hesany et al. (2000), Caudal et al. (2005), Longuet-higgins 


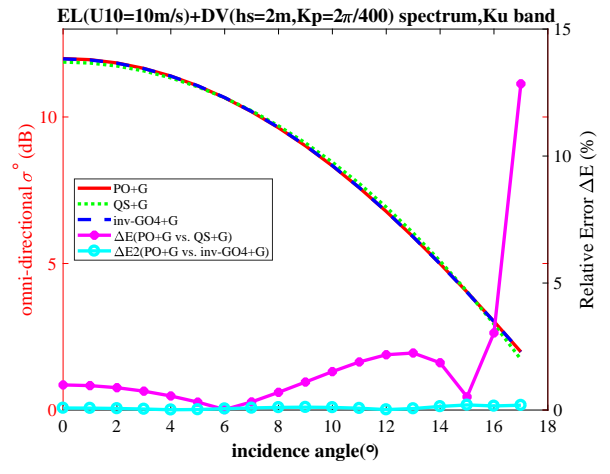

(a)

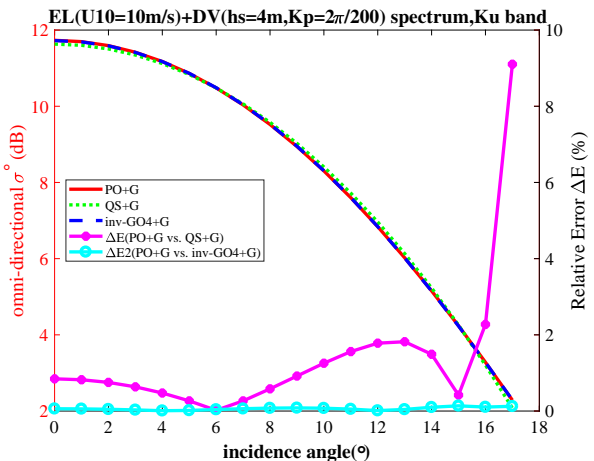

(b)

Fig. 2. Same as in Fig.1 but for mixed sea conditions: (a) El spectrum with $U 10=10 \mathrm{~m} / \mathrm{s}$ combined with swell spectrum with $H_{s}=2 \mathrm{~m}, K_{p}=2 \pi / 400$. (b) El spectrum with $U 10=10 \mathrm{~m} / \mathrm{s}$ combined with swell spectrum with $H_{s}=4 \mathrm{~m}, K_{p}=2 \pi / 200$.

(1982)). Fig. 1 and Fig. 2 present $\sigma^{\circ}(\theta)$ calculated with PO for Ku-band and considering the anisotropic Gaussian case for the surface description. In Fig.1 surface conditions correspond to a pure wind sea case with a $10 \mathrm{~m} / \mathrm{s}$ wind speed and a wave spectrum given by Elfouhaily et al. (1997)-named here after EL. In Fig.2, swell is taken into account in addition, to represent mixed sea conditions. Here, the swell spectrum is defined as proposed by Durden and Vesecky (1985).

$$
\psi(k, \varphi)=F(k) G(\varphi)
$$

$$
F(k)=\frac{H_{s}^{2}}{32 \pi \sigma_{l}^{2}} \exp \left[-\frac{1}{2}\left(\frac{k-k_{\text {peak }}}{\sigma_{l}}\right)^{2}\right], \quad G(\varphi)=\frac{\cos ^{14}\left(\varphi-\varphi_{0}\right)}{\int \cos ^{14}\left(\varphi-\varphi_{0}\right) d \varphi}
$$

where $H_{s}$ is the significant wave height of the swell, $K_{\text {peak }}$ is the peak wave number of the swell, $\sigma_{1}$ the spectral width (fixed as $\sigma_{l}=0.006 \mathrm{rad} / \mathrm{m}$ ). For Fig.2(a), we chose $H_{s}=2 \mathrm{~m}$, $K_{\text {peak }}=2 \pi / 400 \mathrm{rad} / \mathrm{m}$, while for Fig. $2(\mathrm{~b}), H_{s}=4 \mathrm{~m}, K_{\text {peak }}=2 \pi / 200 \mathrm{rad} / \mathrm{m}$.

The $\sigma^{0}(\theta)$ values plotted in Fig.1 and Fig.2 represent $\sigma^{0}(\theta)$ obtained as averaged values of individual values $\sigma^{0}(\theta, \varphi)$ calculated over all azimuths $\varphi$. The light blue curve represent the results obtained by fitting the GO4 shape of Eq.(2) to the PO model (in all azimuths and then averaging), denoted by inv-GO4. The blue curve represents $\sigma^{0}(\theta)$ of QS calculated 
by fitting QS of Eq.(4) to PO. All these curves scale with the left axis. The relative errors between different methods (inversed GO4, inversed QS) and the PO model are also plotted with the right axis scale as reference. We define the relative error between PO and other models as:

$$
\operatorname{err}(\theta)=\frac{1}{N} \sum_{i=1}^{N}\left|\frac{\sigma_{p o}^{0}\left(\theta, \phi_{i}\right)-\sigma_{\bmod }^{0}\left(\theta, \phi_{i}\right)}{\sigma_{p o}^{0}\left(\theta, \phi_{i}\right)}\right|
$$

Where $N$ represents the number of azimuth angles in $0-360^{\circ}$ for the same incidence angle, $\sigma^{0}(\theta, \varphi)$ is in $\mathrm{dB}$ units.

The results show that for the three surface conditions illustrated here, the relative error between the fitted GO4 $\sigma^{\circ}(\theta)$ and the PO values stay close to zero over the incidence range of $0-17^{\circ}$ (and a wind of $10 \mathrm{~m} / \mathrm{s}$ ). The errors for the QS inversion are larger than those for inv-GO4 all over the $0-17^{\circ}$ incidence range; for the incidence less than $15^{\circ}$, they stay of the order of $1 \%$, but increase rapidly with incidence, reaching more than $12 \%$ for incidence $17^{\circ}$. At $6^{\circ}$ and $15^{\circ}$, the error approaches zero because the result of the fit of the QS model to the $\mathrm{PO}$ value (in $\mathrm{dB}$ ) results in two crossing points of the curves close to these incidences. In contrast the error of inversion with GO4 (inv-GO4) stays under $0.19 \%$ for all incidence angles shown. Fig. 2 also shows that taking into account swell in addition to wind sea (for a wind of $10 \mathrm{~m} / \mathrm{s}$ ) does not change significantly the shape nor amplitude of $\sigma^{\circ}(\theta)$, compared to the pure wind sea case (Fig.1). For the same wind speed, when a swell with a $2 \mathrm{~m}$ significant wave height is added in the simulation (Fig.2a), the errors of the inv-GO4 model and inverted QS model with respect to PO are almost not changed. But the addition of a swell with a larger significant wave height (4 $\mathrm{m}$ in Fig.2b), makes the error of the QS model reduce to about $9 \%$, and that of inv-GO4 to $0.12 \%$ at the incidence of $17^{\circ}$. For both cases of mixed sea condition, the inversion with GO4 provides values much closer to PO than does the inversion with QS.

Using the GO4 model to fit $\sigma_{\circ}$ values simulated with the PO model under anisotropic Gaussian assumptions for the sea surface, we hence show that in Ku-band GO4 can reproduce PO with the accuracy as high as the order of $0.2 \%$ for all incidence angles below $15^{\circ}$. This 
high accuracy makes it possible to invert the high order statistics of the quasi-Gaussian sea surfaces from the $\sigma^{0}(\theta, \varphi)$ profiles measured by Ku-band radar with low incidences as it will be shown in Section 4.

In appendix, the same type of analysis is presented for other radar wavelengths (in $\mathrm{C}$ and Ka-band). It is found that with increasing radar frequency (from about 5 to $14 \mathrm{GHz}$ ) the performance of the QS model respect to PO, increases whereas the performance of GO4 does not change significantly. This is because for QS model only the filtering effect is taken into account, whereas for GO4 model both curvature and filtering effects are taken into account. When the electromagnetic frequency is not very high, such as in C-band, ignoring the curvature effect leads to a decreased accuracy of the QS model, whereas the GO4 model which accounts for curvature effects keeps a good accuracy. When the electromagnetic frequency increases, the conditions are closer to the optical limit and the curvature effect are weaker for the short scales. So, with increasing frequency the accuracy of QS gets better whereas that of GO4 stays almost constant.

Before using GO4 for inversion, it is necessary to define the interval of validity in terms of radar geometry and surface conditions. As mentioned in Section 1, the final goal in our study is to invert quasi-Gaussian slope statistical parameters for sea surfaces, especially the higher order statistics, such skewness and peakedness coefficients. Because high order statistics have a weak effect on the backscattering coefficients, an EM model with a high accuracy is required to invert these statistics in order to avoid that the error of the EM model itself contaminates the inversion. Therefore, we consider that the EM model is relevant only when its error is small, e.g., below $0.2 \%$ with respect to PO.

The relative error between GO4 is defined as $\Delta E=\frac{1}{N M} \sum_{i=1}^{N} \sum_{j=1}^{M} \frac{\left|\sigma_{G O 4}^{0}\left(\theta_{i}, \varphi_{j}\right)-\sigma_{P O}^{0}\left(\theta_{i}, \varphi_{j}\right)\right|}{\left|\sigma_{P O}^{0}\left(\theta_{i}, \varphi_{j}\right)\right|}$. Where $N$ and $M$ are the number of incidence and azimuth angles considered in the inversion. $\sigma^{0}(\theta, \varphi)$ is in $\mathrm{dB}$ units. Table 1 shows the relative error $(\Delta \mathrm{E}(\%))$ between GO4 inverted $\sigma^{\circ}$ and PO values in the case of pure wind sea (EL spectrum) for different incident ranges and for different wind speed $2-18 \mathrm{~m} / \mathrm{s}$.

From Table 1, it is seen that a larger range of incidence range leads to larger errors. This 
Table 1: the relative error $(\Delta \mathrm{E}(\%))$ between inv-GO4 and PO

\begin{tabular}{|c|c|c|c|c|c|c|c|c|c|}
\hline wind speed $(\mathrm{m} / \mathrm{s})$ & 2 & 4 & 6 & 8 & 10 & 12 & 14 & 16 & 18 \\
\hline$\Delta \mathrm{E}\left(0-12^{\circ}\right)$ & 0.17847 & 0.00451 & 0.00168 & 0.00667 & 0.04478 & 0.06859 & 0.07658 & 0.07829 & 0.07730 \\
\hline$\Delta \mathrm{E}\left(0-13^{\circ}\right)$ & 0.21292 & 0.00447 & 0.00545 & 0.00852 & 0.05275 & 0.09238 & 0.10654 & 0.11016 & 0.10929 \\
\hline$\Delta \mathrm{E}\left(0-14^{\circ}\right)$ & 0.30127 & 0.01184 & 0.01494 & 0.01014 & 0.06046 & 0.11855 & 0.14260 & 0.14967 & 0.14948 \\
\hline$\Delta \mathrm{E}\left(0-15^{\circ}\right)$ & 0.95597 & 0.05047 & 0.03868 & 0.00997 & 0.06409 & 0.14735 & 0.18414 & 0.19748 & 0.19910 \\
\hline$\Delta \mathrm{E}\left(0-16^{\circ}\right)$ & 2.31700 & 0.36057 & 0.12206 & 0.01285 & 0.06763 & 0.17732 & 0.23681 & 0.25810 & 0.26176 \\
\hline$\Delta \mathrm{E}\left(0-17^{\circ}\right)$ & 4.21928 & 0.55997 & 0.36373 & 0.04344 & 0.08787 & 0.20484 & 0.29885 & 0.33748 & 0.34734 \\
\hline$\Delta \mathrm{E}\left(0-18^{\circ}\right)$ & 6.54778 & 1.26956 & 0.56968 & 0.26304 & 0.11783 & 0.22935 & 0.36916 & 0.43413 & 0.45499 \\
\hline
\end{tabular}

increased errors is linked to the basic approximation of the GO4 formulation (Boisot et al. (2015)) to approximate the PO model. Indeed this approximation is less and less valid when the Rayleigh parameter increases, and this latter decreases with incidence as it involves the electromagnetic wavenumber projected on the vertical axis.

One can choose the incidence range and wind speed range for the inversion by GO4 by setting a threshold on the inversion error. Here the accuracy threshold is set as $0.2 \%$. Table. 1 shows that for wind speed from 4 to $18 \mathrm{~m} / \mathrm{s}$, and incidence ranges of $0-12^{\circ}$ to $0-15^{\circ}$, the relative errors $\Delta \mathrm{E}$ remain smaller than $0.2 \%$. For very low wind speed $(2 \mathrm{~m} / \mathrm{s})$ and larger incidence range $\left(0-16^{\circ}, 0-17^{\circ}, 0-18^{\circ}\right), \Delta \mathrm{E}$ are beyond $0.2 \%$. From these simulation results, it appears that for inversion with GO4, data should be limited to wind speeds within the 4-18 $\mathrm{m} / \mathrm{s}$ range and incidence angle below $15^{\circ}$.

We have also calculated the errors between inv-GO4 values and PO values in the case of a surface described by a mixed wave spectrum (EL+DV), and reached the same conclusion.

The next step for analyzing the conditions of applications of the GO4 is to assess the domain of wavelength representative of the inverted parameters. In the following, without losing the general properties of the GO4 model, we consider that slope and curvature parameters as well as the $R$ parameter may be filtered parameters -also named effective parametersand by using our simulation cases, we examine to which extent this is true.

In the isotropic Gaussian case (Eq.1) these effective parameters are noted $R_{e}, m s s_{e}$ and 
$m s c_{e}$, respectively and $m s s_{e}$ and $m s c_{e}$ are defined from the wave number spectrum as:

$$
\begin{aligned}
m s s_{e} & =\int_{0}^{k d} k^{2} \psi(\vec{k}) d \vec{k} \\
m s c_{e} & =\int_{0}^{k d} k^{4} \psi(\vec{k}) d \vec{k}
\end{aligned}
$$

where the integral are truncated to an upper limit of wavenumber $k d$ in Hauser et al. (2008); Thompson et al. (2005). For the anisotropic and non-Gaussian case (Eq.2) the filtered quantities are:

$$
\begin{aligned}
& m_{s s x_{e}}=\int_{0}^{k d} k_{x}^{2} \psi(\vec{k}) d \vec{k}, \text { mssy }_{e}=\int_{0}^{k d} k_{y}^{2} \psi(\vec{k}) d \vec{k} \\
& m s c x_{e}=\int_{0}^{k d} k_{x}^{4} \psi(\vec{k}) d \vec{k}, m s c y_{e}=\int_{0}^{k d} k_{y}^{4} \psi(\vec{k}) d \vec{k} \\
& m s c x y_{e}=\int_{0}^{k d} k_{x}^{2} k_{y}^{2} \psi(\vec{k}) d \vec{k} \\
& m s s_{e}=\int_{0}^{k d} k^{2} \psi(\vec{k}) d \vec{k}=m s s x_{e}+m s s y_{e} \\
& m s c_{e}=\int_{0}^{k d} k^{4} \psi(\vec{k}) d \vec{k}=m s c x_{e}+m s c y_{e}+2 m s c x y_{e}
\end{aligned}
$$

In order to estimate the limit wave number value $k d$ corresponding to the inverted mean square slope and curvature parameters, we performed a series of inversion of the GO4 model by fitting GO4 to $\sigma^{\circ}(\theta, \varphi)$ values generated with the PO model. Inversion were applied over $\sigma^{\circ}$ profile limited to the incidence range of $\left[0-15^{\circ}\right]$, for wind speeds between 4 and $16 \mathrm{~m} / \mathrm{s}$. The outputs of the fitting process are the slope and curvature parameters of as well as the $R$ coefficient. The method of inversion is non-linear least-square minimization algorithm (as further used for real data inversion, see section 3).

The results for the isotropic case are plotted in Fig.3. The results for the anisotropic case are plotted in Fig.4 and Fig.5. In each case, the parameters inverted by fitting Eq.(1) or Eq.(2) to simulated PO values at C-band (cyan), Ku-band (green) and Ka-band (megenta) are compared on the same figures with the mean square slope and curvature calculated with (Eq.12) and the EL spectrum truncated at a value of $k d$ chosen such that the difference between the two curves (from inversion and from Eq.12) is minimum. We found that this 
corresponds to $k d=68 \mathrm{rad} / \mathrm{m}$ (blue line), $k d=192 \mathrm{rad} / \mathrm{m}$ (red), $k d=513 \mathrm{rad} / \mathrm{m}$ (black), for $\mathrm{C}, \mathrm{Ku}$ and Ka-band respectively. In the same figure the slope or curvature variances for the EL spectrum with $k d=\infty$ (green dashed-dotted line) and $k d=16.5 \mathrm{rad} / \mathrm{m}$ (green dashed line) are also shown. These latter curves correspond approximately to the cases of Cox and Munk clean and slick sea surfaces, respectively in Wu (1972).

Fig.3(a) indicates that the mss obtained by fitting GO4 to PO, exhibit values intermediate between the CM slope variances of clean and slick sea surfaces. The inverted mss increases when the frequency increases. This shows that the inversion provides filtered mss. Indeed the clean sea case of CM in Cox and Munk (1956, 1954), corresponds to $k d=\infty$ since light scattering is sensitive to waves of all scales, whereas the slick case corresponds to $k d=16.5 \mathrm{rad} / \mathrm{m}$ (minimum wavelength of about $38 \mathrm{~cm}$ ) in Wu (1972). For Ku-band, the wave length is about $2.2 \mathrm{~cm}$, thus, $k d$ is in the middle of the values corresponding to Cox and Munk clean and slick sea cases.

Fig.3(a) and (b) show that the omnidirectional curvature variances msc inverted from GO4 have almost the same cutoff wave numbers as those for omnidirectional slope variances: kd $192 \mathrm{rad} / \mathrm{m}$ for Ku-band, $68 \mathrm{rad} / \mathrm{m}$ for C-band and $513 \mathrm{rad} / \mathrm{m}$ for Ka-band. The order of msc magnitude is smaller than that of Boisot et al. (2015). It is because filtered mss as taken into account in our GO4 compensates the curvature effects in the model whereas mss in Boisot et al. (2015) are considered as total.

The results for non-isotropic case are shown in Fig.4 and Fig.5. The directional slope variances $m s s x, m s s y$, and curvature variances $m s c x, m s c y, m s c x y$ have also almost the same cutoff wave numbers as those for omnidirectional slope variances. Thus, we can confirm that all the inverted slope and curvature variances GO4 are filtered, and have a unified cutoff wave number for all parameters at a given frequency. For our inversion conditions (incidence range $0-15^{\circ}$ ), the cutoff wavelength is $1.65,1.48$ and 1.41 times the wavelength of the electromagnetic wave, at $\mathrm{C}, \mathrm{Ku}$ and Ka-band, respectively.

We also examined the effect of the incidence angle on this estimation of the cutoff wavenumber/wavelength. When varying the range of incidence used in the inversion from 


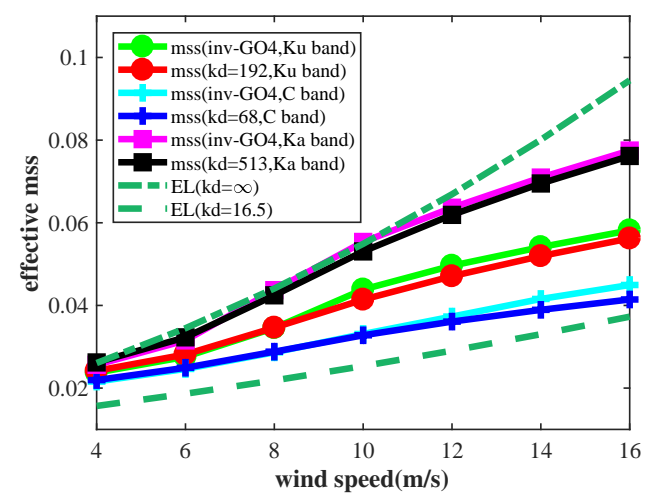

(a)

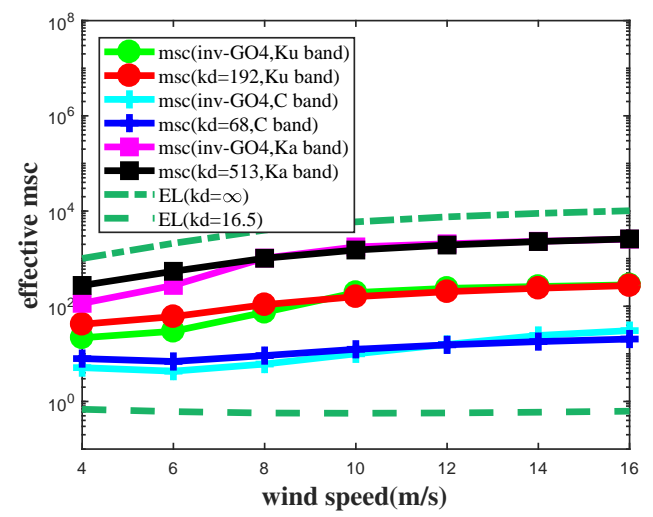

(b)

Fig. 3. Slope variances mss (Fig.3a) and curvature variances msc (Fig.3b) inverted using GO4 shape model fitted on $\mathrm{PO}$ simulated values of $\sigma^{0}(\theta)$ over the incidence range $\left[0-15^{\circ}\right]$ in the isotropic case. The PO values were simulated using the EL spectrum and wind speeds from 4 to $16 \mathrm{~m} / \mathrm{s}$. Results of inversion are shown for C-band (cyan), Ku-band (green) and Ka-band (magenta). Mss calculated with Eq. (12) with $k d=68 \mathrm{rad} / \mathrm{m}, k d=192 \mathrm{rad} / \mathrm{m}, k d=513 \mathrm{rad} / \mathrm{m}$ are shown in blue, red and black respectively. $M s s$ values for $k d=\infty$ (optical limit on clean sea) and $k d=16.5 \mathrm{rad} / \mathrm{m}$ (slick sea case of Cox and Munk) are shown with the dashed dotted and dashed green lines, respectively

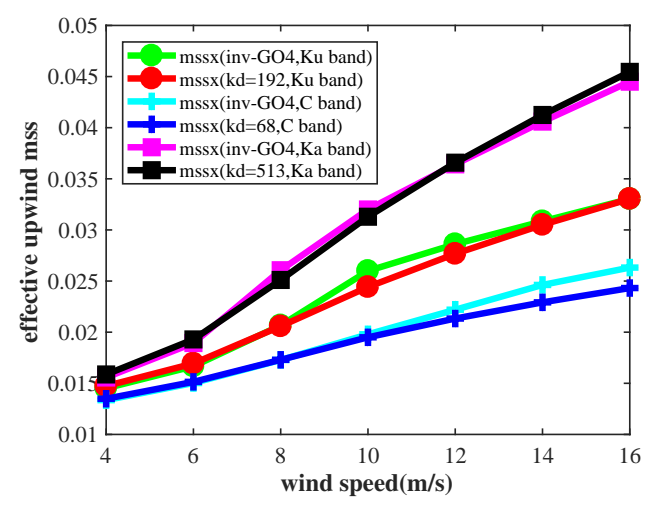

(a)

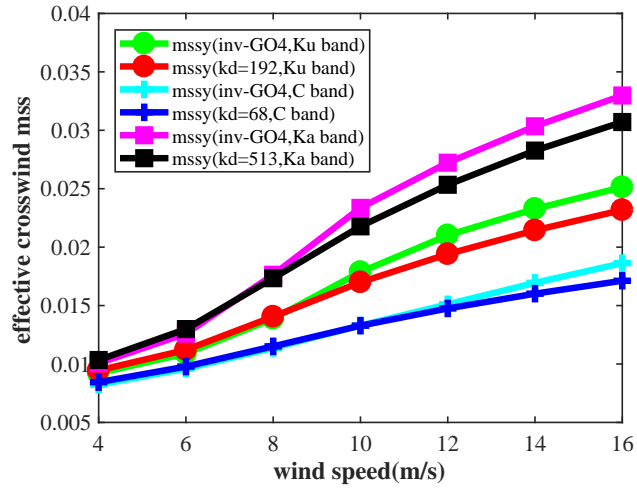

(b).

Fig. 4. Upwind (a) and Crosswind (b) slope variances inverted using GO4 shape model fitted on PO simulated values of $\sigma^{0}(\theta, \varphi)$ over the incidence range $\left[0-15^{\circ}\right]$. The $\mathrm{PO}$ values were simulated using the EL spectrum and wind speeds from 4 to $16 \mathrm{~m} / \mathrm{s}$. Color codes and symbols are the same as in Fig.3. 


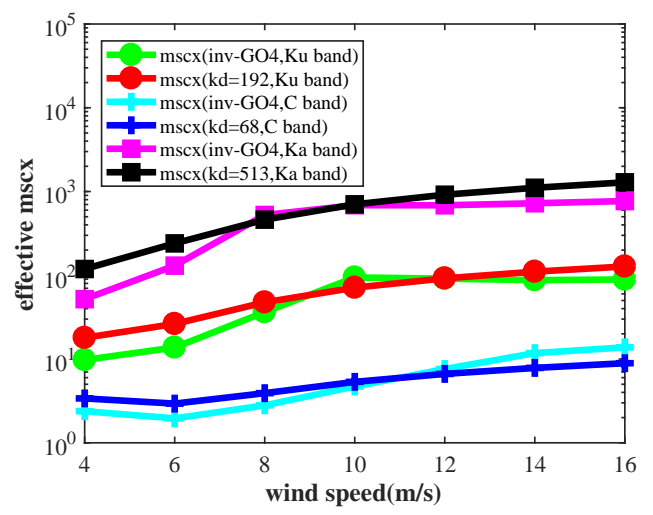

(a)

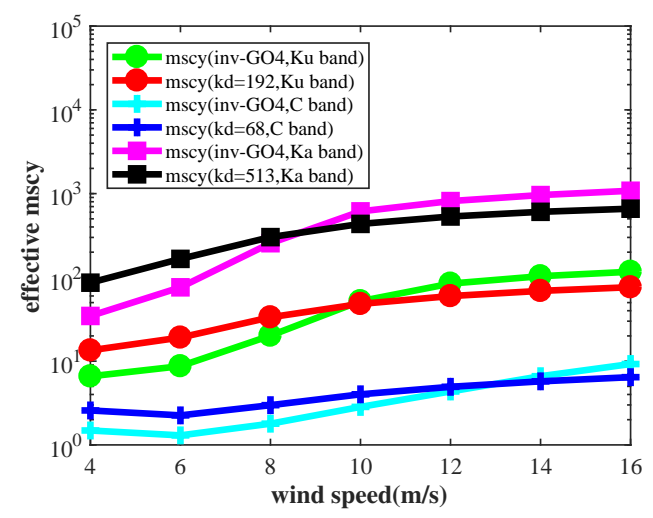

(b)

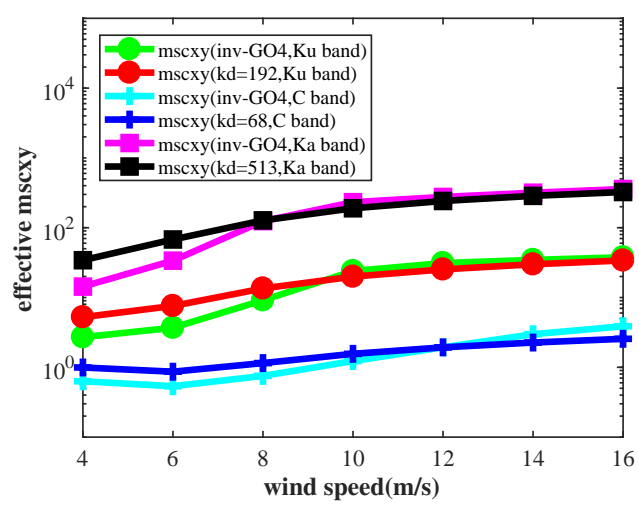

(c)

Fig. 5. As in Fig.4, but for the mean square curvatures mscx, mscy and mscxy

$0-12^{\circ}$ to $0-18^{\circ}$, in the $\mathrm{Ku}$-band case, $k d$ increases from $174 \mathrm{rad} / \mathrm{m}$ to $210 \mathrm{rad} / \mathrm{m}$; the corresponding cutoff wavelength value changes from 1.66 to 1.36 times the wavenumber of the electromagnetic wave.

Fig. 6 shows the $8^{\text {th }}$ parameter inverted in our approach, namely the effective reflection coefficient $R_{e}$. For the three frequencies, $R_{e}$ inverted are smaller than the values of $R$, the theoretical Fresnel Reflection calculated at normal incidence from Klein and Swift (1977) at the temperature of $10^{\circ} \mathrm{C}$ with a salinity of $0.35 \%$.

This means that the inverted parameter $R$ is indeed a kind of diffraction-modified Fresnel coefficient due to the diffraction by waves of very small scales over a surface patch which induces a reflection that is smaller than that by a plane. It is also found that for 4-16 


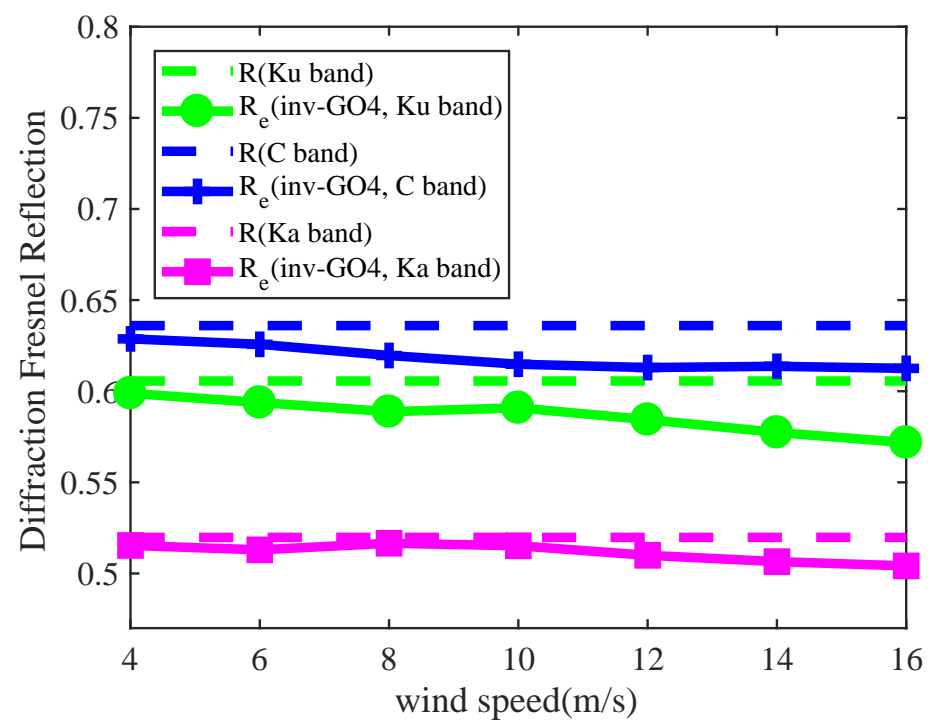

Fig. 6. Inverted Fresnel coefficient. Solid curves are for inverted values, dashed lines are for the theoretical Fresnel coefficient. Color code is: C-band in magenta, Ku-band in green, Ka-band in blue.

$\mathrm{m} / \mathrm{s}$ this diffraction effect increases with wind speed. Such a trend agrees with the results shown in Fig.6 in Freilich and Vanhoff (2003). It is noted however that our retrieved values of $R$ from GO4 at Ku-band are larger than those presented in Freilich and Vanhoff (2003) which are derived by using the QS assumption. This indicates that $R_{e}$ in GO4 includes less diffraction effects than QS, because curvature effects are taken into account in the model.

In summary, we have shown with results of simulations presented in Fig.3 to 6, that all the parameters obtained by inversion of GO4 are filtered quantities. In other words, only the sea waves whose wavelength are greater than a certain threshold (cutoff wavelength) contribute to the backscattering coefficient represented by the GO4 model.

\section{Data}

To invert sea slope PDF, we use HH-polarized $\sigma^{\circ}$ data from the Precipitation Radar (PR) of the TRMM satellite mission (Center (2001)).

PR on board the TRMM satellite is a microwave radar which provides the backscattering coefficients at near-nadir incidence angles ( 0 to $18^{\circ}$ from nadir). The PR antenna is an active 
phased array system of 128 units. Each line consists of 49 pixel angles, and covers ground incidence angle in the across-track direction from $-18^{\circ}$ to $18^{\circ}$ with respect to nadir. Each scan line of PR lasts 0.6 seconds, and the data are obtained with a resolution of $0.1^{\circ}$ in incidence. The backscattering coefficient (NCRS) data of PR are provided after a strict internal and external calibration. The data product used in this paper is PR standard product 2A21 (version-6) from the Distributed Active Archive Center. Nine years of data (2001-2009) of PR surface normalized radar cross-section have been selected over sea under no-rain conditions.

It is known that the inversion of the 2-D slope PDF needs 2-D backscattering coefficients. However, PR only provides 1-D backscattering with incident angles scanned across-track. Here, it is assumed that the parameters of the slope PDF are only related to the wind speed (as it is assumed in Brèon and Henriot (2006); Chu et al. (2012a)), so that the normalized radar cross-section corresponding to a same wind speed at different space or time, can be combined to construct data sets of normalized radar cross-section versus two variables (incidence and direction with respect to the wind direction).

The wind data are provided by the buoy measurements of the National Data Buoy Center (NDBC) from NOAA. They are located in the Atlantic, Pacific, Gulf of Mexico and Caribbean Sea (same data set as used by Chu et al. (2012a)). The different NDBC buoys measure the wind speed at different heights. Here, all buoy wind speeds were normalized to an equivalent anemometer height of $10 \mathrm{~m}$ with the same parameters set as used in Chu et al. (2012a).

We re-use here the same co-located dataset as Chu et al. (2012a), where 82666 matching units are obtained (co-location criterium is a $50 \mathrm{~km}$ diameter area). This corresponds to 15774898 co-located pairs of wind and radar cross-section values. Please refer to the Appendix I in Chu et al. (2012b) for the construction of the collocated dataset in detail.

The co-located data are sorted by wind speed. Because the accuracy of wind speed is 2 $\mathrm{m} / \mathrm{s}$, we have binned the NCRS data at the middle of the wind speed interval (for example $9 \mathrm{~m} / \mathrm{s}$ for all wind speeds from $8 \mathrm{~m} / \mathrm{s}$ to $10 \mathrm{~m} / \mathrm{s}$ ). Fig.7(a) shows the number of data for 


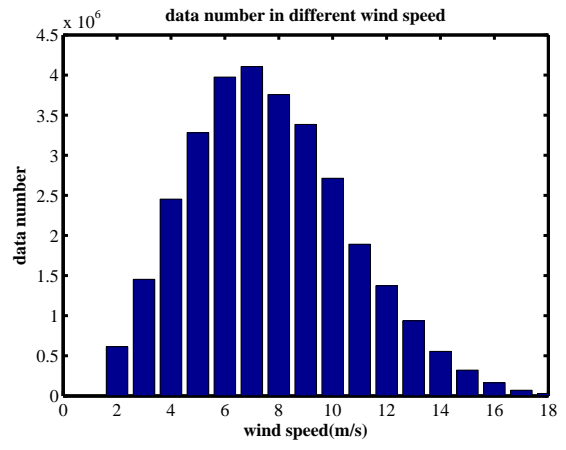

(a)Data number under different wind speed

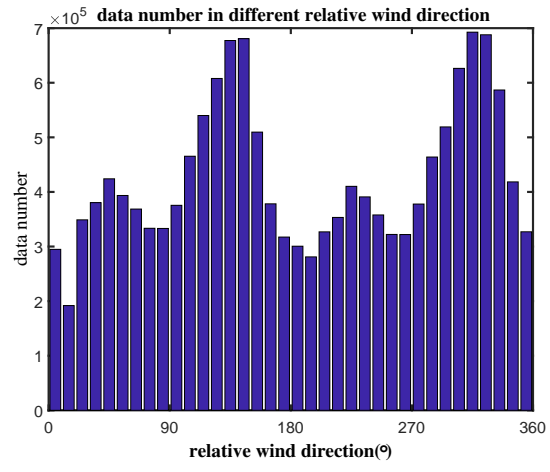

(b)Data number under different relative wind direction.

Fig. 7. Matching data number under different wind speed and different relative wind direction.

different wind speeds, and Fig.7(b) shows the number of data for different relative wind direction. The data are mainly distributed over wind speeds from 2 to $16 \mathrm{~m} / \mathrm{s}$. Based on the results presented in section 2, we have limited our analysis to the wind speed range of 4 to $16 \mathrm{~m} / \mathrm{s}$.

To investigate the impact of sea states on quasi-Gaussian PDF slope, we distinguish two categories of sea states. Using the criteria presented in (A1) of Chu et al. (2012a), we sorted the ocean waves into pure wind sea and dominant swell cases (which can be mixed sea cases).

\section{Inversion Method}

Before discussing the inversion method, the incidence range for the inversion has to be examined again. In section 2, the limit on the incidence range was discusses from the accuracy of GO4 model inversion compared to the PO model. Table 1 shows that this accuracy remains lower than $0.2 \%$ for incidence ranges up to $15^{\circ}$ for wind speeds larger than $4 \mathrm{~m} / \mathrm{s}$. Apart from the accuracy of GO4, the sensitivity of the backscattering to the quasi-Gaussian slope PDF parameters also need to be considered for the choice of the incidence angle range. Direct modeling of $\sigma^{\circ}$ as proposed in Ping Chen and Huang (2015) for the QS case, shows that a variation in peakedness will more significantly affect medium incidence angles than incidence angles very close to nadir. Thus, in order to efficiently invert the peakedness coefficients from 
the $\sigma^{\circ}$ profile, the largest possible range of incidence angle should be chosen. Based on the two above constraints, the range of the incidence $0-15^{\circ}$ is chosen as an appropriate trade-off for the inversion by GO4.

In the anisotropic and quasi-Gaussian case, for a given wind speed, the normalized radar cross-section is dependent on eleven parameters (Eq. (8)) among which seven parameters describe the surface slope PDF $\left(m s s x_{e}, m s s y_{e}, \lambda_{12}, \lambda_{30}, \lambda_{22}, \lambda_{40}, \lambda_{04}\right)$, three parameters are related to curvature variances $\left(m s c x_{e}, m s c y_{e}, m s c x y_{e}\right)$ and the latter is the diffractionmodified reflection coefficient $R_{e}$. In order to estimate the slope PDF parameters in the anisotropic case, a method based on the 2-D backscattering coefficients (i.e., described as a function of incidence and azimuth angles) is required.

For convenience, we transform Eq.(8) into the following form:

$$
\left.\begin{array}{r}
\sigma_{G O 4}^{0}(\theta, \varphi)=\frac{\left|R_{e}\right|^{2}}{2 \sqrt{m s s x_{e}} \sqrt{m s s y_{e}}} \sec ^{4}(\theta) \exp \left(-\frac{1}{2}\left(X^{2}+Y^{2}\right)\right) \times \\
1+\left[\begin{array}{l}
\frac{1}{4} \lambda^{\prime}{ }_{22} H_{2}(X) H_{2}(Y) \\
+\frac{1}{24} \lambda^{\prime}{ }_{40} H_{4}(X) \\
+\frac{1}{24} \lambda^{\prime}{ }_{04} H_{4}(Y)
\end{array}\right] \\
+\frac{1}{6}\left[3 \lambda_{12} H_{1}(X) H_{2}(Y)+\lambda_{30} H_{3}(X)\right]
\end{array}\right\}
$$

Where

$$
\begin{aligned}
& \lambda_{22}^{\prime}=\frac{m s c x y_{e}}{Q_{z}^{2} m s s x_{e} \cdot m s s y_{e}}+\lambda_{22}, \lambda^{\prime}{ }_{40}=\frac{m s c x_{e}}{Q_{z}^{2} m s s x_{e}^{2}}+\lambda_{40} \\
& \lambda_{04}^{\prime}=\frac{m s c y_{e}}{Q_{z}^{2} m s s y_{e}^{2}}+\lambda_{04}
\end{aligned}
$$

It is found that the form of Eq.14 for GO4 is the same as Eq.9 for QS for a quasi-Gaussian sea surface except that $\lambda_{22}^{\prime}, \lambda_{40}^{\prime}$ and $\lambda_{04}^{\prime}$ in Eq.9 are replaced by $\lambda_{22}^{\prime}, \lambda_{40}^{\prime}$, and $\lambda_{04}^{\prime}$ in Eq.14. The parameters $\lambda_{22}^{\prime}, \lambda_{40}^{\prime}$, and $\lambda_{04}^{\prime}$ are the sum of two terms. For an example $\lambda_{22}^{\prime}$, is the sum of $\lambda_{22}$, and of term related to the curvature (curvature term). So if one wants to use QS model directly to invert $\lambda_{22}$, then the inverted $\lambda_{22}$ is not the real peakedness coefficient, but a coefficient contaminated by the curvature effect. This curvature term in each expression of Eq.15 is a small correction which involves a ratio of large quantities ( $m s c x_{e}$, $m s c y_{e}$ or $m s c x y_{e}$ and $Q_{z}{ }^{2}$ ), as well as small quantities $m s s x_{e}, m s s y_{e}$ in denominator. Taking 
$m s s x_{e}, m s s y_{e}, m s c x_{e}, m s c y_{e}$ and $m s c x y_{e}$ in the curvature term as parameters to be inverted simultaneously with the other parameters of Eq.14 is subject to large errors. Thus, instead of inverting those parameters in the curvature terms simultaneously with the PDF coefficients, we directly calculate the curvature terms with Eq.13 and the EL wind sea spectrum of the corresponding known wind speeds and $k d$. We checked that when using the $m s s x_{e}$, mssye, $m s c x_{e}, m s c y_{e}$ and $m s c x y_{e}$ values for the mixed wind sea and swell (EL/DV spectrum) case, the obtained curvature terms are very close to those of the wind sea case (EL spectrum), with difference smaller than $1 \%$.

Then finally, the eight inverted parameters are $R_{e}, m_{s s x_{e}}, m s s y_{e}, \lambda_{12}, \lambda_{30}, \lambda_{22}, \lambda_{40}$ and $\lambda_{04}$ are obtained by fitting Eq.14 to the 2-D $\sigma^{0}(\theta, \varphi)$ measurements over the chosen range of incidence angles $0-15.1^{\circ}$ (PR incidence angle nearest to $15^{\circ}$ ) and over all azimuth angle $0-360^{\circ}$. The non-linear inversion is based on the minimization of the mean squared difference between the measured $\sigma^{0}(\theta, \varphi)$ and GO4 model values expressed in $\mathrm{dB}$ where this cost function sums for each wind speed class, all the values over the incidence and azimuth angles. This non-linear least-square minimization requires initial values of $R_{e}, m s s x_{e}, m_{s s y_{e}}$ that we set as the results obtained by fitting $\mathrm{PR} \sigma^{0}(\theta, \varphi)$ to QS model for a Gaussian sea surface (Eq.4), it also requires initial values for $\lambda_{12}, \lambda_{30}, \lambda_{22}, \lambda_{40}$ and $\lambda_{04}$ that we set as the values proposed by Cox and Munk (1954). Finally, the seven parameters: $m s s x_{e}, m s s y_{e}, \lambda_{12}$, $\lambda_{30}, \lambda_{22}, \lambda_{40}$ and $\lambda_{04}$ of the slope PDF, are obtained.

Here, the non-linear least square inversion algorithm does not use the approximation of $\log (1+\mathrm{t}) \approx \mathrm{t}$ when $t$ is a small quantity, which was the approximation used by Cox and Munk (1954, 1956). According to Cox and Munk (1954), this approximation causes inherent errors of the order of $10 \%$. But in fact it is dependent of $t$ which is a complex combination of several parameters. We could checked with numerical tests on PDF inversion with and without this approximation, for an example of a wind of $10 \mathrm{~m} / \mathrm{s}$, that the linearization of the PDF proposed by Cox and Munk may induce a bias of up to about $15 \%$ on $\lambda_{12}, 25 \%$ on $\lambda_{22}$ and even more than $100 \%$ on $\lambda_{40}$. The error on the other parameters is less than $10 \%$.

It is also noted that because $R_{e}$ is one of our inverted parameters, any overall calibration 
error in the radar measurement will be reflected in $R_{e}$, so that the PDF inverted parameters depend only on the shape of $\sigma^{\circ}(\theta, \varphi)$, and not on the absolute values of $\sigma^{\circ}(\theta, \varphi)$. This means that potential error on radar calibration will have no important effect on the PDF inversion.

\section{Inversion Result}

We co-located in time and space the PR data sets of nine years (2001-2009) with the corresponding buoy measurements. Then, based on the data set, we inverted the seven parameters of the quasi-Gaussian PDF using the GO4 model and method presented here above.

To evaluate the inversion performance, the relative inversion error is defined as err $=$ $\frac{1}{N \cdot M} \sum_{i=1}^{N} \sum_{j=1}^{M}\left|\frac{\sigma_{P R}^{0}\left(\theta_{i}, \varphi_{j}\right)-\sigma_{G O 4}^{0}\left(\theta_{i}, \varphi_{j}\right)}{\sigma_{G O 4}^{0}\left(\theta_{i}, \varphi_{j}\right)}\right|$ where $\sigma^{0}\left(\theta_{i}, \phi_{j}\right)$ is in dB units.

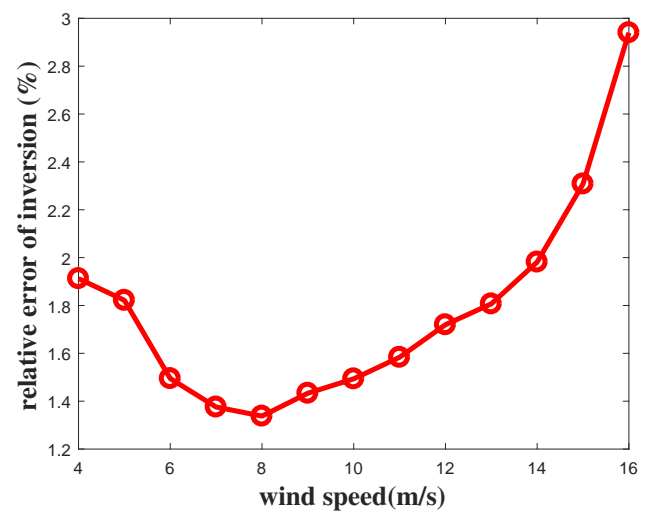

Fig. 8. Relative inversion error under different wind speeds.

Fig.8 shows the inversion error under different wind speeds. At low wind speeds, the errors decreases with wind speed and reaches a minimum at a wind speed of $8 \mathrm{~m} / \mathrm{s}$; for wind speeds larger than $8 \mathrm{~m} / \mathrm{s}$ the error increases with wind speed. The inversion error trend with wind speed is consistent with Table 1 (section 2) which shows the mean difference between GO4 and PO model. In addition, larger errors at wind speeds of 13 to $16 \mathrm{~m} / \mathrm{s}$ may also be attributed to a smaller number data in these conditions, as shown in Fig.7(a). 


\subsection{Second Order Statistical Properties-Slope Variance}

Fig.9(a) shows the filtered (effective) omnidirectional $m s s_{e}$ as a function of wind speed, obtained by fitting Eq.(14) to the $\operatorname{PR~} \sigma^{\circ}(\theta, \varphi)$ data in the incidence angles of $0-15.1^{\circ}$, under conditions of dominant swell (magenta open circles), pure wind wave (green circles) and all cases (red line). Error bars around $m_{s} s_{e}$ for all cases show the effect of changing by \pm 0.7 degree the incidence angle interval considered in the inversion. The figure also shows a comparison with the results of Cox and Munk (1956, 1954) for clean and slick sea surface (dashed-dotted and dashed curves, respectively), the results obtained by Freilich and Vanhoff (2003) (black open squares), and the results calculated with Eq.(13) and the EL spectrum limited to $k d=192 \mathrm{rad} / \mathrm{m}$ (blue line). Fig.9(b)(c) show similarly the upwind $m s s x_{e}$ and crosswind mssye as a function of wind speed.

The general trend of the inverted mss with wind speed is similar to the logarithmic relationship proposed by $\mathrm{Wu}$ (1972). It exhibits values and trend intermediate between the CM slope variances of clean and slick sea surfaces. As shown above, the cutoff wavenumber $k d$ corresponding to the $\mathrm{PR}$ analysis is also about $192 \mathrm{rad} / \mathrm{m}$, which is similar to the simulation result discussed in section 2. Fig.9(a) also shows that the slope variances $m s s_{e}$ are larger than those of Freilich and Vanhoff (2003) by about 20\%-30\%. This is because the slope variances of Freilich and Vanhoff are inverted using the QS model and Gaussian slope PDF, and their results correspond to $k d=50-70 \mathrm{rad} / \mathrm{m}$ by Chu (2011); Freilich and Vanhoff (2003).

From Fig.9, it is shown that adding swell mainly affects the crosswind mss which are slightly higher in swell conditions (Fig.9c). Although not visible in the figures, we confirmed however that mss, mssx and mssy for mixed cases with wind sea and swell are larger than those for pure wind sea. All these results on the effect of the sea conditions on slope variances are consistent with those obtained by Chu et al. (2012a)

We also compared the slope variances in crosswind and upwind obtained with our approach with those obtained by Chu et al. (2012a) and found that ours are both larger by about 20\%-30\% than theirs (not shown). When adding the slope variances in crosswind and upwind of Chu et al. (2012a), we re-produce their total slope variances and find that they 


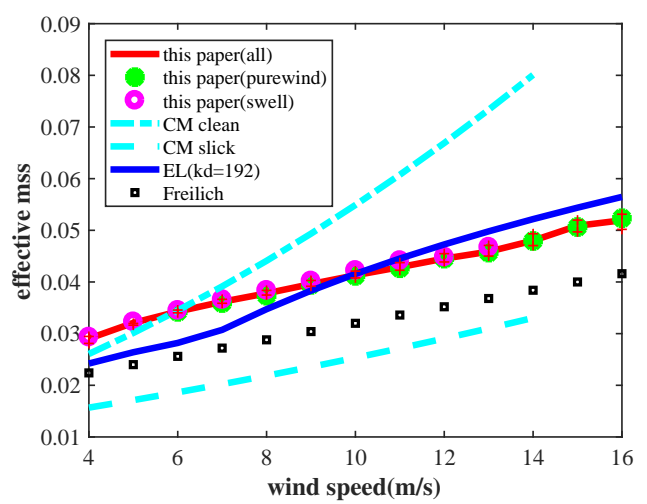

(a) Effective omnidirectional mss

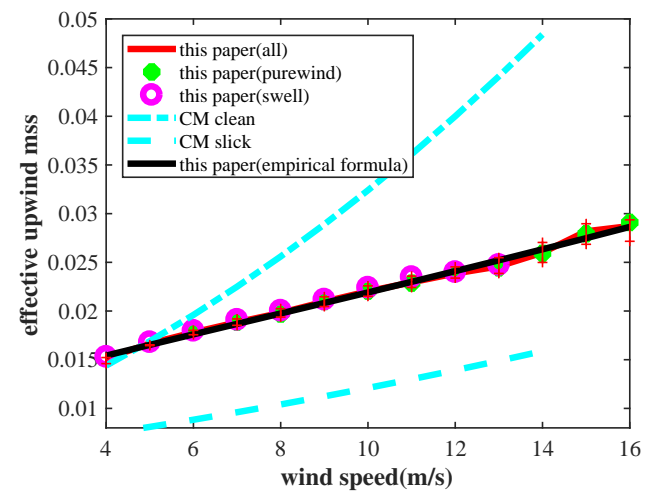

(b) Effective mss(upwind)

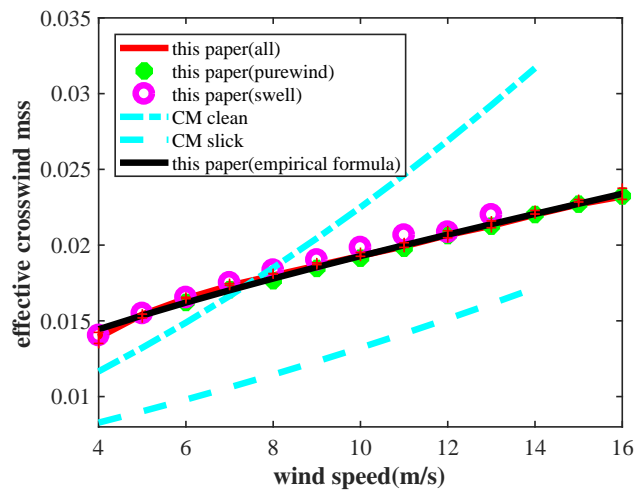

(c) Effective $m s s$ (crosswind).

Fig. 9. Inverted slope variance: effective omnidirectional $m s s$ (a), effective upwind $m s s(\mathrm{~b})$ and effective crosswind mss (c) as function of wind speed. Magenta open circles, green dots and red curve represent the results inverted by fitting GO4 to PR data under conditions of dominant swell, pure wind waves and all cases with incidence angle range $0-15.1^{\circ}$, respectively. Error bars show the effect of changing by $\pm 0.7^{\circ}$ the incidence angle interval considered in the inversion (only visible at the highest wind speeds). In (a), black open squares represent the results obtained by Freilich and Vanhoff (2003). Dashed-dotted and dashed curves correspond to the results of Cox and Munk for clean and slick sea. Cyan lines represents the results calculated with Eq.(13) and the EL spectrum truncated at $k d=192 \mathrm{rad} / \mathrm{m}$. In (b) and (c), the results calculated with the empirical formula Eq.(16-a) and (16-b) are also plotted by black thick lines. 


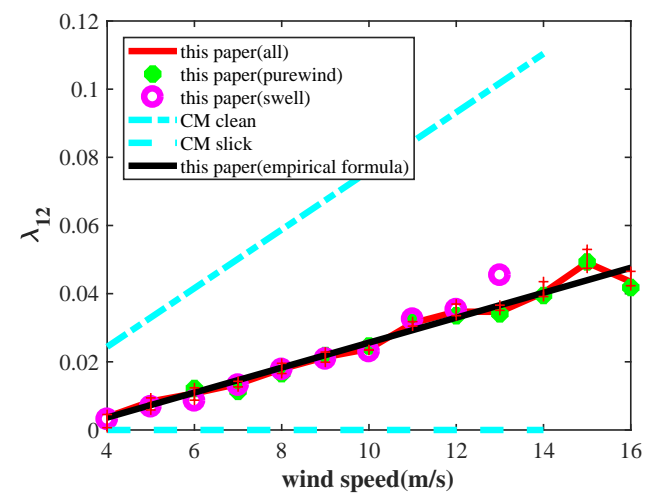

(a)Relationship of skewness coefficient $\lambda_{12}$ with wind speed

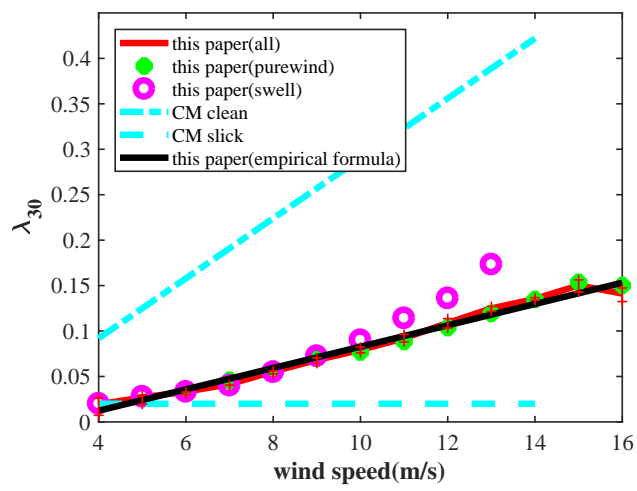

(b)Relationship of skewness coefficient $\lambda_{30}$ with wind speed.

Fig. 10. Inverted skewness coefficients as functions of wind speed for (a) $\lambda_{12}$ (b) $\lambda_{30}$. Color codes and symbols are similar to Fig.9.

Fig.10(a),(b) show skewness coefficients $\lambda_{12}$ and $\lambda_{30}$ as a function of wind speed obtained from inversion of the PR data by fitting GO4. Color codes and symbols are the same as in Fig.9(b). It can be observed that skewness $\lambda_{12}$ and $\lambda_{30}$ inverted from PR data exhibit values intermediate between those of $\mathrm{CM}$ for the two cases (clean and slick sea). This may be attributed to the fact that skewness coefficients are dominated by the shortest waves. Indeed, with a cutoff limit of the GO4 model of about $3.2 \mathrm{~cm}$ (see section 2), the retrieved skewness coefficients are lower than those of all scale waves observed by CM for clean sea, and higher than those of the waves with the cutoff wavelength of $38 \mathrm{~cm}$ observed by $\mathrm{CM}$ for slick sea surfaces in Wu (1972).

Fig.10 also shows that skewness coefficients $\lambda_{12}$ and $\lambda_{30}$ increase with wind speed. This tendency agrees with CM results for a clean sea, with the results by Chu et al. (2012a) and by Brèon and Henriot (2006). $\lambda_{12}$ and $\lambda_{30}$ inverted by our method are a little bit larger than Chu's values (not shown here). This may result from the difference in the numerical method (Chu used the approximation $\log (1+\mathrm{t}) \approx \mathrm{t}$, which causes errors inherent to the numerical inversion as discussed in section 4.) 
Fig. 10(a-b) also shows that sea state conditions significantly affect the skewness coefficient mainly at wind speeds above $10-11 \mathrm{~m} / \mathrm{s}$ where both skewness coefficients are larger in dominant swell conditions than in pure wind sea conditions.

This is consistent with the results of Chu et al. (2012a), who found that $\lambda_{12}$ and $\lambda_{30}$ under dominant swell are larger than those under pure wind waves.

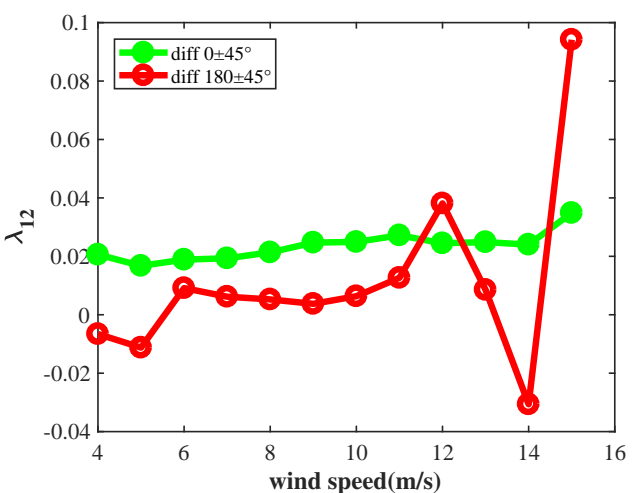

(a) Effect of wind vs. wave direction on skewness coefficient $\lambda_{12}$

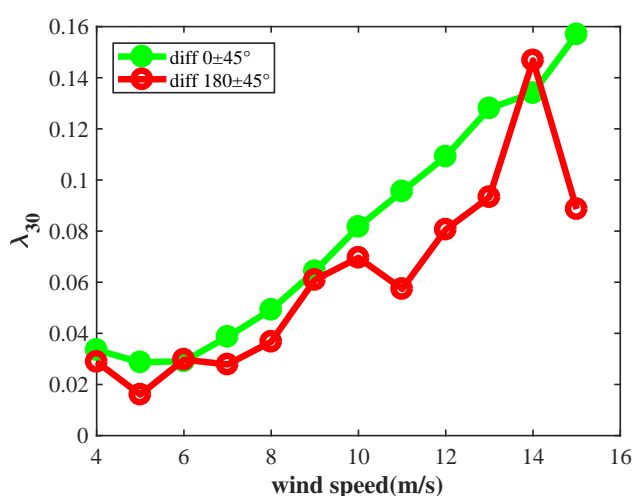

(b) Effect of wind vs. wave direction on skewness coefficient $\lambda_{30}$.

Fig. 11. Effect of wind vs. wave direction on skewness coefficients (a) for $\lambda_{12}$, (b) for $\lambda_{30}$.

We also studied the effect of wind vs. wave direction on skewness coefficients. Fig.11 shows the skewness coefficients as a function of wind speed for two categories of angle between wind and wave directions, the first one for waves more or less parallel to the wind $\left(0^{\circ} \pm 45^{\circ}\right.$, green), the second for waves opposite to the wind $\left(180^{\circ} \pm 45^{\circ}\right.$, red). From Fig.11(a-b) it is found that for waves propagating along-wind and moderate winds (up to $11 \mathrm{~m} / \mathrm{s}$ ), both $\lambda_{12}$ and $\lambda_{30}$ are larger than in the case of opposite waves. At larger winds the number of data sets with opposite waves is relatively small. Therefore, the inversion errors for these cases are larger than $4 \%$. So we can only conclude that for wind speed between 4 and $11 \mathrm{~m} / \mathrm{s}$, $\lambda_{12}$ and $\lambda_{30}$ are larger in cases of wind and waves aligned compared to cases where they are opposite. This may be explained by the fact that waves whose direction is not aligned with the wind direction will decrease the asymmetry of sea surface slope in upwind and downwind directions. 


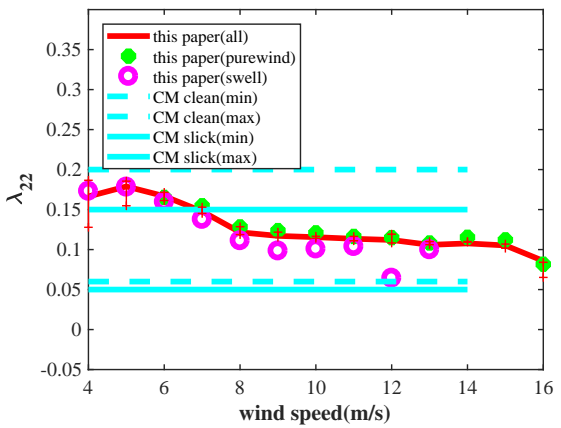

(a) Relationship of peakedness coefficient $\lambda_{22}$ with wind speed.

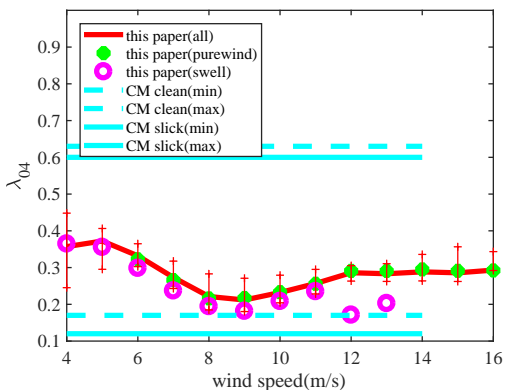

(b) Relationship of peakedness coefficient $\lambda_{04}$ with wind speed.

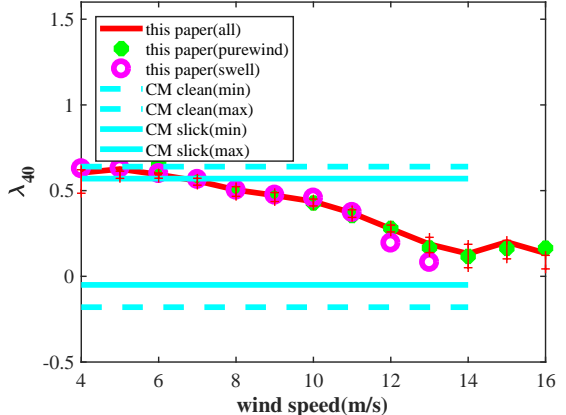

(c) Relationship of peakedness coefficient $\lambda_{40}$ with wind speed.

Fig. 12. Inverted peakedness coefficients as functions of wind speed.

Fig. 12(a)(b)(c) show the results for the peakedness coefficient $\lambda_{22}, \lambda_{04}$ and $\lambda_{40}$ where color codes and symbols are the same as in Fig. 9(b).

In Fig. 12, over the whole wind speed conditions, our values are within the limits found by CM for each case (clean and slick sea). Thanks to our non-linear inversion (in opposite to the case of $\mathrm{CM}$ ) we are able to bring more details on the peakedness parameters. In particular, we find that decreases $\lambda_{40}$ with wind speed up to $14 \mathrm{~m} / \mathrm{s}$ and then remains constant. $\lambda_{22}$ and $\lambda_{04}$ tend to decrease with wind speed up to a wind speed of $8 \mathrm{~m} / \mathrm{s}$ and then remain stable $\left(\lambda_{22}\right)$ or increase slightly $\left(\lambda_{04}\right)$. In opposite, Cox and Munk could only provide a large range of possible values without possibility to identify significant difference between clean and slick sea cases nor trends with wind speed. 
Fig.12(a)(b) also show that the presence of the swell tends to induce smaller values of the peakedness coefficients and at least for wind conditions larger than $6 \mathrm{~m} / \mathrm{s}$ for $\lambda_{22}$ and $\lambda_{04}$ for largest than $11 \mathrm{~m} / \mathrm{s}$ for $\lambda_{40}$.

So peakedness effect seem to be less sensitive to wind speed than skewness coefficients (see above) and less sensitive to the presence of swell. Their tendency to decrease with wind speed in light to moderate winds while staying more or less stable for higher winds may be attributed to a smaller uniformity of the wave slope distribution along the long wave profiles at light winds, according to the phenomenological model proposed by Chapron et al. (2000). The same interpretation might be raised to explain the smaller values of peakedness coefficients when swell is present.

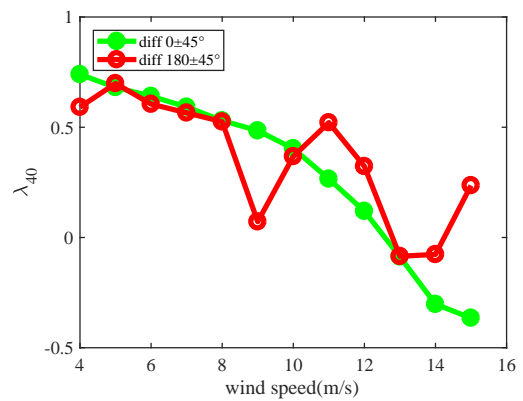

Fig. 13. Effect of wind vs. wave direction on peakedness coefficient $\lambda_{40}$

We also studied the effect of wind vs. wave direction on peakedness coefficients. Fig.13 shows the peakedness coefficient $\lambda_{40}$ as a function of wind speed for two categories of angle between wind and wave directions (similar to the categories in Fig.10). In opposite to the results on skewness shown here above, we find that the angle has no clear effect on $\lambda_{40}$. We also analyzed the peakedness coefficients $\lambda_{22}$ and $\lambda_{04}$ with the same categories of wind/wave angles (not shown here) and draw the same conclusion as for $\lambda_{40}$.

\subsection{Sensitivity to the choice of the angular domain}

To assess our results we also studied the effect of the incidence range on the inversion of the slope pdf parameters. For that purpose, 6 incidence ranges for PR data $\left(0-12.8^{\circ}, 0-13.5^{\circ}\right.$, $\left.0-14.3^{\circ}, 0-15.1^{\circ}, 0-15.9^{\circ}, 0-16.5^{\circ}\right)$ were tested for the inversion, one by one. We found that 
the values of mss, mssx and mssy increase with the incidence range; the increments are within about $10 \%$ for the incidence range from $0-14.3^{\circ}$ to $0-16.8^{\circ}$. This is because when increasing the range of incidence angle, while remaining in quasi-specular conditions, the radar backscatter is more sensitive to short scales waves, that correspond larger slopes. With the incidence ranges increasing from $0-14.3^{\circ}$ to $0-15.9^{\circ}$, the skewness coefficient $\lambda_{30}$ increases by about $10 \%$ for the wind speeds larger than $8 \mathrm{~m} / \mathrm{s}$, while $\lambda_{12}$ decreases with the incidence range from $0-14.3^{\circ}$ to $0-16.8^{\circ}$ for the wind speed between $6 \mathrm{~m} / \mathrm{s}$ to $15 \mathrm{~m} / \mathrm{s}$. For the smaller incidence range, such as $0-12^{\circ}, 0-12.8^{\circ}$ the inverted peakedness coefficients $\lambda_{22}, \lambda_{40}$ and $\lambda_{04}$ have a divergence. It may be due to the fact that for the small incidence range the sensitivity of radar backscattering to the peakedness coefficients is weak (Chu (2011)). The divergence reduces rapidly with increasing incidence range, and disappears starting from the $0-14.3^{\circ}$ range. When varying the incidence range from $0-14.3^{\circ}$ to $0-16.8^{\circ}$, the peakedness coefficients change by about $10 \%$. Overall the conclusions on the trend with wind speed, presence of swell and relative wave directions do not change when varying the incidence range from $0-14.3^{\circ}$ to $0-16.8^{\circ}$.

\subsection{Empirical Formulae}

Based on the relationships of the seven parameters of quasi-Gaussian slope PDF with wind speed from $4 \mathrm{~m} / \mathrm{s}$ to $16 \mathrm{~m} / \mathrm{s}$, based on the inversion results for the incidence range $0-15^{\circ}$ (solid red curves) shown in Fig.8-11, we propose empirical formulae, for quasi-Gaussian sea slope parameters corresponding to a cutoff limit of $192 \mathrm{rad} / \mathrm{m}$ (associated with Ku-band observations from $0-15^{\circ}$ incidence). In this process, we use some analytical shapes proposed in past study, such as a logarithmic dependence with wind speed for the slope variances (Hauser et al. (2008); Wu (1972)), and linear relationships for skewness coefficients with wind speed as proposed by Cox and Munk (1956). In spite of the trend of the three peakedness coefficients with wind speed shown in Fig.12, we still use the linear fit for peakedness 
coefficients. We obtained the following empirical formulae:

$$
\begin{aligned}
& \text { mss }_{e}=0.009416 \times e^{\left(0.2188 \times U^{0.5868}\right)} \pm 0.0041 \\
& \text { mssy }_{e}=0.007392 \times e^{\left(0.3895 \times U^{0.3911}\right)} \pm 0.0027 \\
& \lambda_{12}=0.003663 \times U-0.01101 \pm 0.0139 \\
& \lambda_{30}=0.01174 \times U-0.03462 \pm 0.0443 \\
& \lambda_{40}=-0.04646 \times U+0.8565 \pm 0.1786 \\
& \lambda_{22}=-0.006796 \times U+0.1944 \pm 0.0276 \\
& \lambda_{04}=-0.004321 \times U+0.3273 \pm 0.0466
\end{aligned}
$$

Note that the mean values of our inverted peakedness coefficients $\left(\lambda_{22}=0.1265 \pm 0.0276\right.$, $\lambda_{40}=0.3919 \pm 0.1786$ and $\left.\lambda_{04}=0.2841 \pm 0.0466\right)$ agree well with those given by Brèon and Henriot (2006) that were obtained from optical data; this indicates that the peakedness coefficients can be inverted correctly from Ku-band radar observations using the GO4 model.

\subsection{Slope Probability Density Distribution}

Using the above empirical formulae, valid for $\mathrm{Ku}$-band radar data over the incidence range of $0-15^{\circ}$. the sea surface slope PDF can be obtained for different the wind speeds in the range $4-16 \mathrm{~m} / \mathrm{s}$.

Fig.14(a) shows the slope PDF $p(\tan (\theta), 0)$ or a wind speed of $10 \mathrm{~m} / \mathrm{s}$ in upwind and downwind direction, obtained from the PR data inversion with the quasi-Gaussian PDF (blue curve), from CM for a clean sea (black solid curve with open circles) and from CM for a slick sea (black dotted curve with open circles). The horizontal axis is the slope angle $\theta$, where the positive sign is for upwind direction. In the along-wind direction (Fig.14a), the PDF retrieved from our analysis is intermediate between the clean sea and the slick case of Cox and Munk, with higher probability of large slopes than in the slick case but lower probability of large slopes than in the clean case. This is mainly due to the filtering effects 


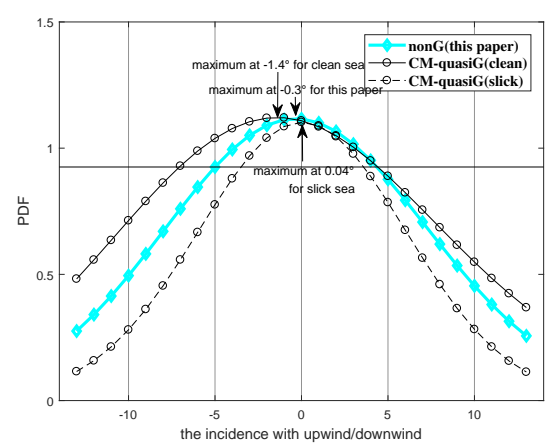

(a) $p(\tan (\theta), 0)$ in upwind direction

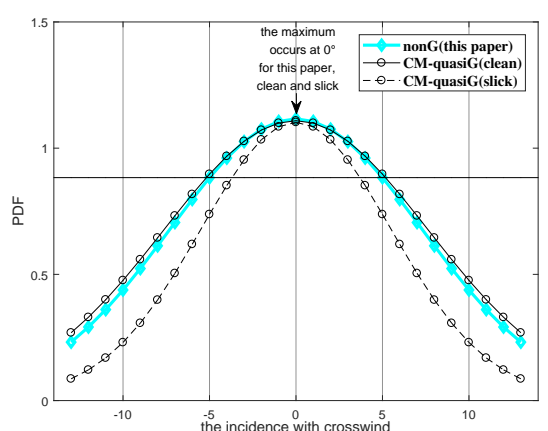

(b) $p(0, \tan (\theta))$ in crosswind direction

Fig. 14. Slope PDF in upwind (a) and crosswind (b) directions, under the condition of a 10 $\mathrm{m} / \mathrm{s}$ wind speed. In (a) the arrows represent the maximum of the $\operatorname{PDF}$ (at $-0.3^{\circ},-1.4^{\circ}, 0.04^{\circ}$ for PR data, CM clean sea and CM slick sea, respectively); in (b) the PDF maximum is at the same position $\left(0^{\circ}\right)$, for the three models.

because waves, which contribute to our analyzed signals are not shorter than $3.2 \mathrm{~cm}$, as shown above. The shape of the along-wind slope PDF is also slightly different because of skewness and peakedness effects. In particular skewness is responsible of the shift of the maximum of the curve with respect to the 0 slopes $\left(-0.3^{\circ}\right.$ for our results compared to $-1.4^{\circ}$ and $0.04^{\circ}$ for respectively the $\mathrm{CM}$ clean sea, and the $\mathrm{CM}$ slick sea cases). This is associated with $\sigma^{\circ}$ values which are with slightly larger at the low incidence angles in downwind direction than in upwind direction. We could confirm this feature by a direct inspection of $\sigma^{\circ}$ variations with azimuth.

Similarly, Fig.14b shows the slope PDF along the crosswind direction $p(0, \tan (\theta))$. The PDF retrieved from our analysis is very close to that corresponding to the clean sea case of Cox and Munk. This was already apparent in Fig.9c with crosswind mss values much closer to the clean sea case than in the case of upwind mss (Fig.9b). The axis of symmetry of the slope PDF is located in the incidence angle of $0^{\circ}$ for all the three cases, e.g. there is no angle deviation for the slope PDF along the crosswind direction. All these features indicate that in the crosswind direction, the slope PDF derived from microwave measurements behave very similarly to the optical case. 


\section{Conclusion}

Up to now only analyses from optical data have provided information on the seven parameters of the quasi-Gaussian wave slope PDF and their relation with wind speed. These results cannot be transposed directly in the application of ocean microwave remote sensing because of the diffraction effects at wavelengths longer than optical ones. In this paper, using a GO4 scattering model and TRMM/PR normalized radar cross-section, we estimate the seven parameters of the quasi-Gaussian wave slope PDF at Ku-band. This is done by applying a nonlinear fit of this model to the 2-D backscattering coefficients (as a function of incidence angle and azimuthal angle with respect to the wind).

In a first step, we checked from simulation performed under a Gaussian assumption and for $\mathrm{Ku}, \mathrm{C}$ and Ka-bands that even if curvature effects are included in GO4, the approach provides filtered variances of slope and curvature, as well as an effective Fresnel coefficient. For a given electromagnetic frequency, the same cutoff was obtained for slope variances and curvature variances. This filtered effect decreases when the electromagnetic wavelength decreases. In our conditions this filtering effect was estimated to be at $3.2 \mathrm{~cm}$, e.g. 1.45 times the electromagnetic wavelength. The slope variances inverted by using the GO4 model are all larger than those inverted by using the Quasi-Specular model, because the curvature effect is taken into account in GO4, which makes more small scale waves being inverted by GO4 than by QS without curvature correction. We also assessed that the optimal range of incidence angles to be used in the inversion with the GO4 model is $0-15^{\circ}$.

Our results obtained by the TRMM/PR data set confirm that the inverted mean square slopes correspond to a filtered surface with filtering effects however less important than when the QS model is used for inversion.

The general trend of mean square slopes retrieved from this analysis is consistent with previous results also obtained in Ku-band (Hauser et al. (2008); Chu et al. (2012a)). One important point to note is that the crosswind mss are closer to the clean sea case of CM than are the alongwind mss.

Concerning the third order statistical properties, we find that skewness coefficients $\lambda_{12}$ 
and $\lambda_{30}$ lie between those of CM for the clean and slick sea conditions and clearly increase with wind speed as found for the optical case by Cox and Munk (1954, 1956); Brèon and Henriot (2006). The existence of swell in addition to wind sea tends to increase the skewness coefficients with respect to cases of pure wind sea, specially at the higher winds. The angle between wave direction and wind direction also affects the skewness coefficients. When waves propagate along the wind direction $\left( \pm 45^{\circ}\right)$ the skewness coefficients $\lambda_{12}$ and $\lambda_{30}$ are larger than when waves propagate in the opposite direction. These results are important because they may explain the trends and part of variability of the upwind to downwind ratio of the backscatter signals in remote sensing.

As for the peakedness coefficients $\lambda_{22}, \lambda_{40}$ and $\lambda_{04}$ inverted by using GO4, they are within the intervals of values found Cox and Munk for all their analyzed sea conditions (clean sea or slick sea). Thanks to our non-linear inversion method without the linearization used by previous authors (Cox and Munk, Chu), the accuracy on the peakedness coefficient is higher so that we could evidence the dependence of the peakedness coefficients with wind speed and sea state conditions. Although they are less variable than the skewness coefficient with wind speed, their tendency may indicate a smaller uniformity of the wave slope distribution along the long wave profiles at light winds compared to moderate or high winds, according to the phenomenological model proposed by Chapron et al. (2000). The same interpretation might be raised to explain the smaller values of peakedness coefficients when swell is present.

In addition, empirical linear models are proposed in this paper for the seven retrieved parameters of the quasi-Gaussian slope PDF as a function of wind speed.

Overall, the slope PDF reconstructed from the microwave observations in Ku-band are either intermediate between those of the optical limit and the slick sea case of Cox and Munk (along wind direction) or very similar to that of the optical limit (crosswind direction).

It should be pointed out that for a given space-time point, the PR radar only provides the 1-D backscattering coefficient as a function of incidence angle cross-track. However, 2-D backscattering coefficients are necessary for a 2-D slope inversion. Therefore in this paper, we have combined the backscatter coefficients corresponding to a same wind speed 
at different space or time to construct 2-D backscattering coefficients for 2-D slope inversion at that wind speed. However, the combinations need the assumption that the slope PDF parameters are only related to the wind speed.

Other kind of radar working at low incidence, wave spectrometer (see e.g., Jackson et al. (1992); Hauser et al. (2008, 1992); Caudal et al. (2014)), which are designed for the measurements of wave directional spectrum, can also measure 2-D scattering coefficient as a function of incident angle and azimuthal angle of $0-360^{\circ}$. In the near future (2018), the SWIM (Surface Waves Investigation and Monitoring) radar, which will be carried on the CFOSAT (China-France Oceanography Satellite) will provide simultaneously the normalized radar backscatter at near-nadir incidence in a 2-D geometry and 2-D spectra of ocean dominant waves. Hence, it will give new opportunities to further study the relationship between the slope PDF parameters and the wind and long waves. Future work using a large data set from satellite should also be used in combination with external data from models or in situ measurements to assess the impact of atmospheric stability on peakedness of the slope PDF (Shaw and Churnside (1997); Longuet-higgins (1982); Mc Daniel (2003)).

The result presented here on the non-Gaussian slope PDF are associated to Ku-band conditions and cannot be generalized to other conditions because of remaining filtering effects which depend in electromagnetic wavelength, even if they are smaller than when using a Quasi-specular model for the inversion. However, the main trends with wind speed and wave conditions found here may be more general since for all parameters, we find trends which are intermediate between the optical limit (Cox and Munk clean sea and slick sea case). Analysis of Ka-band data with the approach proposed here will be of particular interest because we expect to be close to the optical limit where all scales account.

\section{Acknowledgments}

The authors would like to thank Professor Yijun He and Researcher Xiaoqing Chu for providing the PR data set matched with NDBC buoy, and thank Dr. Charles-Antoine Guerin and Dr. F. Nouguier for giving some important suggestions to our work. The 
authors greatly appreciate the anonymous reviewers for their detailed comments that helped to improve the manuscript. This work was supported in part by the National Key Research and Development Program of China (2016YFC1401005), by the Open Fund of the State Key Laboratory of Satellite Ocean Environment Dynamics (SOED1607).

\section{Appendixes}

In order to examine the effect of frequency on the performance of inv-GO4, Fig.A1 and Fig.A2 show $\sigma^{0}(\theta)$ calculated with PO, inv-GO4 and QS for C-band and Ka-band, respectively, with EL spectrum $(U=10 \mathrm{~m} / \mathrm{s})$. Color codes and symbols are the same as in Fig.1.

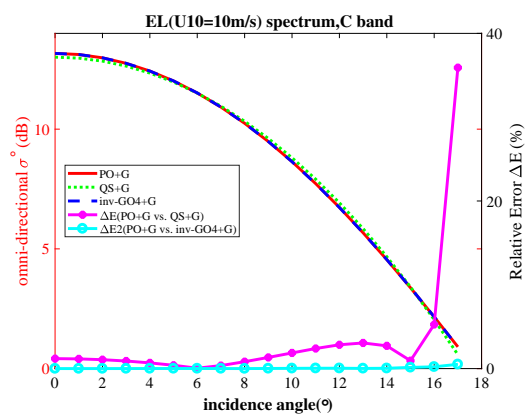

Fig. A1. $\sigma^{0}(\theta)$ (in $\mathrm{dB}$ ) as a function of $\theta$ with the $\mathrm{PO}$ model (red line) for a $10 \mathrm{~m} / \mathrm{s}$ wind speed, using EL spectrum, for C-band. Color codes and symbols are the same as in Fig.1

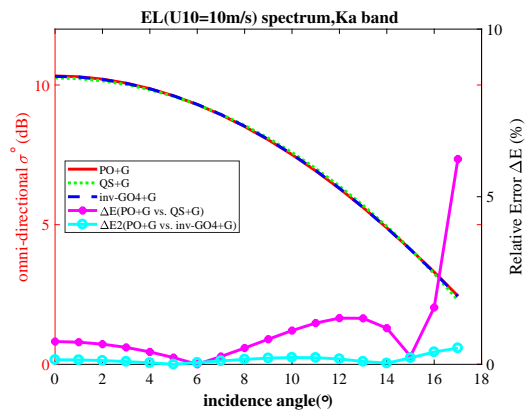

Fig. A2. $\sigma^{0}(\theta)$ (in $\mathrm{dB}$ ) as a function of $\theta$ with the $\mathrm{PO}$ model (red line) for a $10 \mathrm{~m} / \mathrm{s}$ wind speed, using EL spectrum, for Ka-band. Color codes and symbols are the same as in Fig.1 


\section{References}

Barrick, D. E., 1968. Relationship between slope probability density function and the physical optics integral in rough surface scattering. Proceedings of the IEEE 56 (10), 1728-1729.

Boisot, O., Nouguier, F., Chapron, B., Guèrin, C. A., 2015. The go4 model in near-nadir microwave scattering from the sea surface. IEEE Transactions on Geoscience andr Remote Sensing 53 (11), 5889-5900.

Brèon, F. M., Henriot, N., 2006. Spaceborne observations of ocean glint reflectance and modeling of wave slope distributions. Journal of Geophysical Research Oceans 111 (C6), C06005.

Bringer, A., Guerin, C. A., Chapron, B., Mouche, A. A., 2012. Peakedness effects in nearnadir radar observations of the sea surface. IEEE Transactions on Geoscience and Remote Sensing 50 (9), 3293-3301.

Caudal, G., Dinnat, E., Boutin, J., 2005. Absolute calibration of radar altimeters: Consistency with electromagnetic modeling. Journal of Atmospheric and Oceanic Technology $22(6), 771-781$.

Caudal, G., Hauser, D., Valentin, R., Gac, C. L., 2014. Kuros: A new airborne ku-band doppler radar for observation of surfaces. Journal of Atmospheric and Oceanic Technology 31 (10), 2223-2245.

Center, E. O., 2001. TRMM data users handbook. National Space Development Agency of Japan.

Chapron, B., Kerbaol, V., Vandemark, D., Elfouhaily, T., 2000. Importance of peakedness in sea surface slope measurements and applications. Journal of Geophysical Research Oceans 105 (C7), 17195C17202.

Chu, X., 2011. The algorithm and applied basic study on remote sensing of ocean wave spectrum for ocean wave spectrometer. Ph.D. thesis, Chinese Academy of Sciences. 
Chu, X., He, Y., Chen, G., 2012a. Asymmetry and anisotropy of microwave backscatter at low incidence angles. IEEE Transactions on Geoscience and Remote Sensing 50 (10), 4014-4024.

Chu, X., He, Y., Karaev, V. Y., 2012b. Relationships between ku-band radar backscatter and integrated wind and wave parameters at low incidence angles. IEEE Transactions on Geoscience and Remote Sensing 50 (11), 4599-4609.

Cox, C., Munk, W., Nov 1954. Measurement of the roughness of the sea surface from photographs of the sun's glitter. J. Opt. Soc. Am. 44 (11), 838-850.

Cox, C. S., Munk, W. H., 1956. Slopes of the sea surface deduced from photographs of sun glitter. Bull.scripps Inst.oceanogr 6.

Durden, S. L., Vesecky, J. F., 1985. A physical radar cross-section model for a wind-driven sea with swell. IEEE Journal of Oceanic Engineering 10 (4), 445-451.

Elfouhaily, T., Chapron, B., Katsaros, K., Vandemark, D., 1997. A unified directional spectrum for long and short wind driven waves 102 (C7), 15.

Freilich, M. H., Vanhoff, B. A., 2003. The relationship between winds, surface roughness, and radar backscatter at low incidence angles from trmm precipitation radar measurements. Journal of Atmospheric and Oceanic Technology 20 (4), 549-562.

Hauser, D., Caudal, G., Guimbard, S., Mouche, A. A., 2008. A study of the slope probability density function of the ocean waves from radar observations 113 (C2), 710-713.

Hauser, D., Caudal, G., Rijckenberg, G. J., Vidal-Madjar, D., Laurent, G., Lancelin, P., 1992. Ressac: a new airborne fm/cw radar ocean wave spectrometer. IEEE Transactions on Geoscience and Remote Sensing 30 (5), 981-995.

Hesany, V., Plant, W. J., Keller, W. C., 2000. The normalized radar cross section of the sea at 10 incidence. Geoscience and Remote Sensing IEEE Transactions on 38 (1), 64-72. 
Hwang, P. A., 2005. Wave number spectrum and mean square slope of intermediate-scale ocean surface waves. Journal of Geophysical Research Oceans 110 (C10), 9.

Hwang, P. A., Wang, D. W., 2004. An empirical investigation of source term balance of small scale surface waves. Geophysical Research Letters 31 (15), 121-141.

Jackson, F. C., Travis, W. W., Peng, C. Y., 1985. A comparison of in situ and airborne radar observations of ocean wave directionality 90 (NC1), 1005andndash;1018.

Jackson, F. C., Walton, W. T., Hines, D. E., Walter, B. A., Peng, C. Y., 1992. Sea surface mean square slope from ku-band backscatter data. Journal of Geophysical Research: Oceans 97 (C7), 11411-11427.

URL http://dx.doi.org/10.1029/92JC00766

Klein, L., Swift, C., 1977. An improved model for the dielectric constant of sea water at microwave frequencies. IEEE Transactions on Antennas \& Propagation 2 (1), 104-111.

Longuet-Higgins, M. S., 1978. On the DynamicsOn the Skewness of Steep Gravity Waves in Deep Water. Springer US.

Longuet-higgins, M. S., 1982. On the skewness of sea-surface slopes. Journal of Physical Oceanography 12 (11), 1283-1291.

Mc Daniel, S. T., 2003. Microwave backscatter from non-gaussian seas. IEEE Transactions on Geoscience and Remote Sensing 41 (1), 52-58.

Om, P., 1985. Spectral and statistical properties of the equilibrium range in wind generated gravity waves. Journal of Fluid Mechanics 156 (-1), 505-531.

Ping Chen, Q. Y., Huang, P., 2015. Effect of non-gaussian properties of the sea surface on the low-incidence radar backscatter and its inversion in terms of wave spectra by an ocean wave spectrometer. Journal of Oceanology and Limnology 33 (5), 1142-1156. 
Resio, D., Perrie, W., 02 1991. A numerical study of nonlinear energy fluxes due to wave-wave interactions part 1. methodology and basic results. Journal of Fluid Mechanics $223(-1)$, 603-629.

Shaw, J. A., Churnside, J. H., Jun 1997. Scanning-laser glint measurements of sea-surface slope statistics. Appl. Opt. 36 (18), 4202-4213.

URL http://ao.osa.org/abstract.cfm?URI=ao-36-18-4202

Thompson, D. R., Elfouhaily, T. M., Garrison, J. L., 2005. An improved geometrical optics model for bistatic gps scattering from the ocean surface. IEEE Transactions on Geoscience and Remote Sensing 43 (12), 2810-2821.

Tsang, L., Kong, J. A., 2001. Scattering of Electromagnetic Waves, Advanced Topics. Wiley.

Vandemark, D., Chapron, B., Sun, J., Crescenti, G. H., Graber, H. C., 2004. Ocean wave slope observations using radar backscatter and laser altimeters. Journal of Physical Oceanography 34 (12), 2825-2842.

Wu, J., 1972. Sea surface slope and equilibrium wind wave spectra 15 (5), 741-747.

\section{List of Figures}

Figure 1: The value of $\sigma^{\circ}(\theta)$ using EL spectrum for Ku-band..................................... 10

Figure 2: the value of $\sigma^{\circ}(\theta)$ using EL spectrum combined with swell spectrum ............ 11

Figure 3: $\quad$ The inverted mss and $m s c$ by inv-GO4 model ........................................ 17

Figure 4: $\quad$ The inverted mss, mssx and mssy by inv-GO4 model ................................. 17

Figure 5: $\quad$ The inverted mscx, mscy and mscxy by inv-GO4 model.............................. 18

Figure 6: The inverted fresnel coefficient by inv-GO4 model .................................... 19

Figure 7: The data number under different wind speed and relative wind direction ...... 21

Figure 8: Relative inversion error under different wind speeds.................................. 24

Figure 9: $\quad$ The inverted mss, mssx and mssy by inv-GO4 model with PR data ............ 26 
841 Figure 10: The inverted skewness coefficients by inv-GO4 model with PR data ............. 27

842 Figure 11: Effect of wind vs. wave direction on skewness coefficients ............................ 28

843 Figure 12: The inverted peakedness coefficients by inv-GO4 model with PR data.......... 29

844 Figure 13: Effect of wind vs. wave direction on peakedness coefficient.......................... 30

845 Figure 14: Slope PDF in upwind and crosswind directions............................................. 33

${ }_{846}$ Figure A1: The value of $\sigma^{\circ}(\theta)$ using EL spectrum for C-band ....................................... 37

${ }_{847}$ Figure A2: The value of $\sigma^{\circ}(\theta)$ using EL spectrum for Ka-band....................................... 37 